\title{
Criminologie
}

\section{Prisons, sida et divulgation de renseignements médicaux. Analyse juridique et éthique}

\section{Ralf Jürgens et Norbert Gilmore}

Volume 27, numéro 2, 1994

Sexe et criminalité

URI : https://id.erudit.org/iderudit/017358ar

DOI : https://doi.org/10.7202/017358ar

Aller au sommaire du numéro

\section{Éditeur(s)}

Les Presses de l'Université de Montréal

ISSN

0316-0041 (imprimé)

1492-1367 (numérique)

Découvrir la revue

Citer cet article

Jürgens, R. \& Gilmore, N. (1994). Prisons, sida et divulgation de renseignements médicaux. Analyse juridique et éthique. Criminologie, 27(2), 127-163.

https://doi.org/10.7202/017358ar
Résumé de l'article

The article addresses some of the issues raised in federal correctional institutions by the generation and communication of personal medical information pertaining the HIV infection. It examines, first, whether medical information pertaining to federal inmates - information considered confidential between medical staff and an inmate - can be disclosed absent the inmate's consent. It then examines what conditions or criteria determine whether or not such disclosure is ethically and legally justifiable, and if disclosure is justified, what conditions apply to its disclosure. Finally, specific situations in which a claim for disclosure may arise are examined.

The article concludes that in federal correctional institutions, the disclosure of personal medical information absent consent of the person is seldom justifiable. In most situations, such disclosure is unnecessary and even appears to be counterproductive or harmful. Measures that can be undertaken to prevent exposure to and infection with HIV have to be undertaken regardless of whether an inmate or staff member is or is not known, to staff, wardens, or inmates, to be infected with HIV. 
The article addresses some of the issues raised in federal correctional institutions by the generation and communication of personal medical information pertaining the HIV infection. It examines, first, whether medical information pertaining to federal inmates - information considered confidential berween medical staff and an inmate - can be disclosed absent the inmate's consent. It then examines what conditions or criteria determine whether or not such disclosure is ethically and legally justifiable. and if disclosure is justified, what conditions apply to its disclosure. Finally, specific situations in which a claim for disclosure may arise are examined.

The article concludes that in federal correctional institutions, the disclosure of personal medical information absent consent of the person is seldom justifiable. In most situations, such disclosure is unnecessary and even appears to be counterproductive or harm- ful. Measures that can be undertaken to prevent exposure to and infection with HIV have to be undertaken regardless of whether an inmate or staff member is or is not known. to staff, wardens, or inmates, to be infected with HIV.

\section{A. INTRODUCTION}

Les questions soulevées par le VIH/sida et l'usage de la drogue dans les prisons préoccupent énormément les détenus, les autorités carcérales et la population. Bon nombre de ces questions sont controversées, et elles ont souvent été au cceur de l'activité des médias et des débats sur la place publique et dans l'arène politique. Parmi les principaux sujets discutés et débattus, il y a lieu de signaler : la prestation de soins de santé : l'éducation des détenus et du personnel au sujet du VIH/sida et de la consommation de drogues; le placement des détenus infectés par le VIH ou atteints du sida; la santé et la sécurité au travail du personnel correctionnel; la disponibilité de condoms. d'aiguilles ou de seringues et du matériel nécessaire permettant le

1. Les auteurs sont respectivement coordonnateur et président du Comité d'experts sur le sida et les prisons. Cet article est une adaptation de plusicurs lextes a paraître dans le Rapport final du Comité, en particulier de l'annexe 7 de la documentation. Les auteurs désirent renercicr le professeur M. A. Somerville pour sa contribution a la rédaction du présent article, et monsieur J. Dussault pour la révision linale du texte. 
nettoyage de ces dernières: l'accès aux tests de dépistage de l'anticorps anti-VIH et à des services de counseling; le traitement des toxicomanes : la confidentialité du dossier médical d'un détenu.

Le nombre de détenus infectés par le VIH ou atteints du sida n'est pas connu, faute de dépistage systématique au sein de la population carcérale. Il n'y a pas de statistiques sur le nombre de détenus infectés par le VIH ou atteints du sida dans les prisons provinciales québécoises. En revanche. dans les pénitenciers fédéraux, au cours de juin 1993, on savait que 95 des 13110 détenus étaient infectés par le VIH, ce qui représente une hausse substantielle par rapport à janvier 1992, où l'on savait que 56 des 12838 détenus étaient infectés, et ce qui indique que, dans les établissements canadiens, le taux global d'infection est de 1 détenu sur $138(0,7 \%)$. Des études menées dans des établissements fédéraux et provinciaux permettent toutefois de penser que l'ampleur du problème de l'infection à VIH dans les établissements correctionnels canadiens serait supérieure à ce que cette statistique pourrait faire croire. Une étude de séroprévalence menée dans un établissement fédéral en Ontario (établissement Joyceville) a révélé un taux d'infection de un pour cent (Ford, 1993). Des études menées dans des prisons provinciales ont révélé des taux d'infection variant entre un pour cent parmi les détenus à leur entrée dans des établissements correctionnels de la Colombie-Britannique et $7,2 \%$ dans un établissement à sécurité moyenne pour femmes, à Montréal (Hankins, 1989). Même s'il ne convient pas d'appliquer les résultats de ces études à d'autres prisons ni à la même prison à une date ultérieure (parce que la composition de la population et les risques de transmission varient selon les établissements et que le roulement des détenus est élevé), ces études révèlent néanmoins un taux d'infection à VIH beaucoup plus élevé que celui de l'ensemble de la population au Canada. Ceci résulte du fait que parmi les détenus des établissements correctionnels canadiens, on retrouve un nombre élevé de personnes qui ont eu des activités à risque élevé de transmission du VIH : l'emprunt ou le prêt de seringues servant à l'injection de drogues et la pratique de relations sexuelles non-protégées pouvant survenir dans le contexte d'un échange contre de la drogue, de la nourriture ou de l'argent. À titre d'exemple, mentionnons que dans l'étude menée dans la prison pour femmes à Montréal, toutes les femmes séropositives étaient des utilisatrices de drogues injectables et que dix d'entre elles avaient déclaré qu'avant leur incarcération, la prostitution était leur principale source de revenus.

Initialement. la réaction au problème soulevé par le VIH/sida dans les prisons canadiennes ne s'est manifestée que très lentement. Peu de solutions furent envisagées afin de développer des politiques ou de mettre sur pied des programmes éducatifs destinés tant au personnel qu'aux détenus. De plus, on ne distribuait aucun condom, aiguille, seringue ou matériel permettant de nettoyer aiguilles et seringues. Au Québec, la Commission 
des droits de la personne avait reçu plusieurs demandes d'enquête de la part de détenus incarcérés dans des établissements de détention provinciaux, alléguant qu'ils subissaient, dans leurs conditions de détention. de la discrimination fondée sur leur séropositivité. Leurs principaux arguments étaient qu'ils ne pouvaient demeurer parmi les autres détenus même si leur santé le leur permettait, mais qu'il se voyaient confinés au service de santé du centre de détention. qu'on leur refusait l'accès au travail rémunéré et qu'on ne respectait pas la confidentialité de leur dossier médical. En 1990, la Commission avait recommandé au ministre responsable des centres de détention d'intervenir sans délai en vue d'assurer aux détenus porteurs du VIH des conditions appropriées à leur état physique. Néanmoins, ce n'est qu'en avril 1992 qu'une politique relative aux maladies infectieuses en milieu carcéral a été adoptée par la Direction de la détention.

En décembre 1991, une étape importante a été franchie en vue de l'adoption de mesures efficaces visant à prévenir la transmission du VIH en milieu correctionnel : à compter de cette date. les condoms furent accessibles aux détenus dans les pénitenciers fédéraux. Par la suite, des établissements de certaines provinces ou territoires ont emboîté le pas, dont le Québec depuis le 16 novembre 1992. Ceci a donné lieu à des discussions plus ouvertes sur la santé des détenus, leur accès aux soins de santé et la prévention des maladies infectieuses dans les prisons.

Dans un effort de trouver des solutions au problème du VIH/sida dans les pénitenciers fédéraux. le solliciteur général du Canada a créé, le 15 juin 1992, un comité d'experts sur le sida et les prisons (CESP). Le comité a pour but d'aider le gouvernement fédéral à promouvoir et à protéger la santé des détenus et du personnel et à prévenir la transmission du VIH et d'autres agents infectieux dans les établissements correctionnels fédéraux. Il a examiné et analysé des questions soulevées par l'infection à VIH. le sida et l'usage de la drogue dans ces établissements, et a tenu des consultations à ce sujet. Au cours de 1992, le CESP a visité des établissements correctionnels de la Colombie-Britannique, de l'Ontario et du Québec, il a examiné et analysé des politiques nationales et internationales, des rapports et des documents pertinents et a demandé à des détenus, à des membres du personnel carcéral ainsi qu'à des groupes et des personnes s'intéressant au $\mathrm{VIH} /$ sida et aux prisons de lui transmettre des renseignements et des rapports ${ }^{2}$.

2. Le Comite va bientôt soumettre ses recommandations dans le rapport du Comité qui sera compose de trois documents: 1 . Le VIH/sida en milieu carcéral: Rapport sommaire et reconmandations, 2. Le VIH/sida en milieu carcéral : Rapport final du Comité d'experts sur le sida et les prisons, sur lequel est londé le rapport sommairc, et Le VIH/sida en milieu carcéral : documentation. 
Le comité a fait face à une tâche difficile : chercher à réduire les risques de transmission de maladies infectieuses telles le VIH/sida dans le milieu complexe et l'atmosphère souvent surchauffée des établissements correctionnels. Cette tâche s'est révélée d'autant plus difficile que ces maladies peuvent être transmises lors de rapports sexuels, qu'on perçoit comme un comportement répréhensible en prison, ou par le partage d'aiguilles pour la consommation de drogues, activité tout aussi condamnable aux yeux de l'État et qui constitue un acte criminel.

Bon nombre des difficultés que soulève le VIH/sida dans les prisons découlent de ce que Harding a défini comme un conflit de valeurs sousjacent entre le système pénal (fondé sur les principes du «juste dû», du rétributivisme et de la dissuasion) et le modèle des soins médicaux. fondé sur la promotion de la santé et la réduction de la souffrance (Harding, 1990). Le secteur correctionnel est axé sur la sécurité du public ou l'application de la loi, et non sur la santé publique (Brewer, 1991). À l'extérieur du cadre carcéral, on a découvert que les interventions coercitives étaient souvent sans effet et risquaient d'être contre-productives en matière de contrôle de la transmission du VIH et de ses conséquences (Somerville. 1989), et que les meilleures interventions étaient celles qui reposaient sur le respect des personnes, de leurs droits et de leur dignité, favorisaient la responsabilité personnelle et offraient des possibilités réalistes d'adopter un comportement sécuritaire. Toutefois, si on veut prévenir les maladies et assurer des soins médicaux en milieu carcéral, il faut concilier un modèle médical fondé sur la prévention, le diagnostic. les soins et les traitements avec les exigences correctionnelles de la détention et du contrôle (Neveloff Dubler. 1990). Les précocupations relatives à la sécurité et l'aspect punitif inhérents au système carcéral peuvent faire sérieusement obstacle à la prévention du VIH/sida dans les prisons. Dans ce milieu, les populations qui ont des comportements à risque agissent souvent en cachette, l'accès aux moyens nécessaires pour prévenir la transmission du VIH est très limité, ce qui complique d'autant les interventions en paroles et en actes propres à corriger la situation.

Pour toutes ces raisons. il est plus difficile qu'ailleurs de réagir à la menace que pose le VIH/sida en milieu carcéral. Mais en même temps, les prisons offrent d'importantes occasions de prévenir la transmission du VIH parce qu'il y passe une proportion importante de gens ayant des activités à risque, en particulier l'usage de drogues injectables (Brewer, 1991).

Le présent article porte sur certaines des questions soulevées dans les établissements correctionnels fédéraux par la production et la communication de renseignements médicaux personnels touchant l'infection à VIH. Les auteurs se sont concentrés sur les établissements correctionnels tédéraux. mais la plupart de leurs analyses et conclusions pourraient également 
s'appliquer dans le cas de prisons provinciales. Dans les pénitenciers, les employés ont souvent prétendu qu'ils ont besoin de connaître l'état sérologique des détenus infectés afin de prendre les précautions nécessaires pour se protéger eux-mêmes ainsi que leurs familles respectives. En premier lieu. les auteurs cherchent à déterminer si les renseignements médicaux sur les détenus de ressort fédéral - renseignements considérés comme confidentiels entre le personnel médical et un détenu - peuvent être divulgués sans le consentement du détenu. Ils examinent ensuite dans quelles conditions et selon quels critères la divulgation serait justifiable du point de vue de l'éthique et du droit. Enfin, ils analysent des situations précises pouvant donner lieu à une demande de divulgation de ces renseignements.

\section{B. ANALYSE DES ASPECTS JURIDIQUES ET ÉTHIQUES DE LA DIVULGATION DE RENSEIGNEMENTS MÉDICAUX}

\section{L'obligation de confidentialité}

\section{Le droit}

En droit, les médecins ont une obligation de confidentialité envers leurs patients (Casswell. 1989). Tous les renseignements résultant d'une relation médecin-patient doivent être tenus confidentiels : le patient y a ơroit et le médecin a cette obligation (Gilmore et Somerville, 1989). Ce principe est reconnu en common law ${ }^{3}$ et est souvent reprise dans les lois ${ }^{4}$. En outre, dans l'arrêt Mclnerney c. MacDonald, la Cour suprême du

3. Casswell (1989), en référence à A.B. v. C.D., (1851) 14 Sess. Cas. (Second Serics) 177 (Scot. Ct. Sess.); Re Inquiry into the Confidentiality of Health Records in Ontario, (1979) 98 D.L.R. (3d) 704, 714, 24 O.R. (2d) 545, 555 (C.A. Ont.), le juge d'appel Dubin, pour la majorité : "Les membres de la profession médicale ont une obligation de confidentialité envers leurs patients. Ils sont tenus de ne donner aucun renseignement touchant l'état de leurs patients ou les services professionnels qu'ils rendent [...]. m(C'est nous qui traduisons.)

4. Pour une liste des lois provinciales reconnaissant l'obligation de confidentialité, voir Casswell (1989), p. 228, note 13 : le Health Disciplines Act Medicine Regulation. R.R.O. 1980, Règlement $n^{\circ} 448$, art. 27.22; la Charte des droits et libertés de la personne, L.R.Q. (1977), chap. C-12, art. 9; la Loi médicale, L.R.Q. (1977), chap. M-9. art. 42, et le Code de déontologie des médecins, L.R.Q. (1981), chap. M-9, r. 4, art. 3.01. Voir également l'arrêt de la Cuur suprême du Canada dans Frenette c. Métropolifaine (La), cie d'assurance-vie, [1992] 1 R.C.S. 647-696, 666: «Au Québec, les dossiers hospitalicrs (art. $7 \mathrm{dc}$ la Loi sur les services de santé et les services sociaux) ainsi que les renseignements tévélés à un médecin en raison de sa profession (deuxième alinéa de l'art. 9 de la Charte el art. 42 de la Loi médicale) jouissent de la protection de la loi qui les place sous le sceau de la confidentialite. Le droit a la non-divulgation de renseignements confidentiels en général est également prévu au premicr alinća de l'art. 9 de la Charte.» Voir aussi p. 673. 
Canada a qualifié de «fiduciaire» la relation médecin-patient et a statué que certains devoirs découlent de cette relation de confiance particulière :

Parmi ceux-ci. il y a le devoir du médecin d'agir en toute bonne foi et avec le plus grand dévouement. et de préserver le caractère confidentiel des renseignements obtenus d'un patient ou à son sujet. [...] Quand un patient communique des renseignements personnels dans le contexte d'une relation médecin-patient, il le fait en s'attendant légitimement à ce que ces devoirs soient remplis. (McInerney c. MacDonald, [1992] 2 R.S.C. 138. p. 149.)

La Cour suprême a poursuivi en disant que, quoique le médecin soit le propriétaire du dossier médical en tant que support, il doit utiliser son contenu pour le profit du patient et que, «comme l'information est confiée au médecin à des fïns médicales. il en résulte une attente de la part du patient qu'il continuera d'avoir un droit et d'exercer un contrôle sur cette information » (lbid., p. 151).

Dans la décision Hay v. University of Alberta Hospital ((1990) 69 D.L.R. (4th) 755 (C.B.R. Alb.), cité dans Frenette c. Métropolitaine (La), cie d'assurance-vie, [1992] 1 R.C.S. 647-696, p. 688), le juge Picard a décrit ainsi le droit à la confidentialité :

La relation entre un médecin et son patient est confidentielle, mais le patient peut renoncer à ce droit à la confidentialité. En effet, la confidentialité constitue un attribut important de la relation entre un médecin et son patient. un attribut essentiel pour favoriser une communication franche entre eux. Le patient peut expressément renoncer à ce droit ou on peut juger qu'il l'a fait implicitement d'après les actes qu'il a accomplis. En d'autres cas, l'existence d'un intérêt public supérieur ou d'une directive législative peut justifier un médecin de divulguer des renseignements sur son patient. En l'absence de ces circonstances, le droit demeure et le médecin qui divulgue des renseignements confidentiels pourrait faire l'objet d'une poursuite pour violation du caractère confídentiel, une possibilité qui suscite manifeste. ment certaines préoccupations chez les médecins (lbid. pp. 757-758; c'est nous qui traduisons.)

Comme l'a déclaré la Cour suprême du Canada, le devoir de préserver la confidentialité des renseignements qui découle de la relation médecinpatient vise à favoriser la divulgation de renseignements par le patient à son médecin et à encourager la communication entre médecin et patient (McInerney, p. 153). Il faut inciter les patients à se taire traiter sans crainte que leur maladie. leur état ou leur traitement soient divulgués. En outre. "le fait d'encourager les patients à se présenter pour être traités profite non seulement aux patients eux-mêmes. mais également à ceux qui peuvent risquer l'intection; c'est dire que conserver la confiance du patient n'est 
pas seulement dans l'intérêt de ce dernier, mais également dans l'intérêt public» (Casswell. 1989, p. 229 : c'est nous qui traduisons.)

L'obligation de confidentialité peut même être garantie par la Constitution ${ }^{5}$. De plus, dans la majorité des provinces et territoires, les médecins sont expressément tenus par la loi de préserver la contïdentialité des renseignements sur les patients, relativement aux maladies transmissibles, y compris le sida 6 .

Au niveau fédéral, la Loi sur la protection des renseignements personnels (L.R.C. (1985), ch. P-21) vise à protéger les Canadiens contre la collecte et la diffusion non justifiées de renseignements personnels à leur sujet par les institutions et les fonctionnaires fédéraux. Mentionnons, entre autres mesures de dissuasion dans ce cas, la responsabilité résultant de la communication injustifiée ou non autorisée et de l'usage abusif de tels renseignements, ainsi que les recours pouvant être exercés en cas d'abus résultant d'une telle communication, par exemple la protection contre la discrimination.

\section{Les obligations éthiques}

Les médecins ont également l'obligation éthique de préserver la confidentialité des renseignements fournis par leurs patients. Cette obligation a son origine dans le serment d'Hippocrate, établi au IV ${ }^{\mathrm{e}}$ siècle avant JésusChrist, qui contient ce vau :

Quoi que je voie ou entende dans la société pendant l'exercice ou même hors de l'exercice de ma profession, je tairai ce qui n'a jamais besoin d'être divulgué, regardant la discrétion comme un devoiren pareil cas. (Le Serment d'Hippocrate, Grand Larousse universel, tome 13, 1985, p. 9513.)

Le Code de déontologie de l'Association médicale canadienne exige que le médecin respecte les confidences reçues de son patient et ne divulgue ces renseignements qu'avec la permission du patient, ou si la loi l'y oblige $^{7}$. Pour ce qui est du VIH/sida, on a dit que «selon un principe général de l'éthique médicale. le médecin qui a connaissance de résultats

5. Casswell (1989), p. 228 , note 14 , référence a Picard, 1984 , p. 8 , note 51 , se reportant à la Charte canadienne des droits et libertés, Loi constitutionnelle de 1982 , partie I, art. 7.

6. Pour un aperçu des lois el règlements qui exigent la confidentialité des renscignements sur Ies patients relativement aux maladies contagieuses, infectieuses, Iransmissibles et à déclaration obligatoire, voir Casswell (1989), p. 255 (tableau A); Hamblin (1990).

7. L'article 6 du Code de déontologie de l'Association médicate canadienne prévoit ce qui suit: «Pour se conformer à la déontologie médicale, le médecin doit : respecter les conlidences reçues de ses malades ou collègues et ne divulguer ces renseignenents qu'avec la permission du malade, saul' lorsque la loi l'y oblige». Cité dans Casswell (1989), 226, note 3. 
positifs ou même d'un diagnostic confirmé de sida doit protéger la confidentialité de ces renseignements et en éviter la communication » (Belitzky et Solomon, 1987 ; c'est nous qui traduisons.)

\section{La politique carcérale}

Le principe général selon lequel les renseignements médicaux sont confidentiels s'applique également aux établissements correctionnels. Les détenus ont les mêmes droits, notamment les droits à l'autonomie, à l'inviolabilité et à la confidentialité, que tous les autres Canadiens, sauf lorsque ces droits sont nécessairement abrogés du fait de l'emprisonnement ${ }^{8}$. Il a été déclaré qu' "à moins d'une preuve claire et convaincante qu'une personne incarcérée est susceptible de transmettre le VIH, la menace générale de la transmission du VIH dans les établissements correctionnels ne peut justifier une recherche obligatoire» (Somerville et Gilmore, 1988; c'est nous qui traduisons). Cette conclusion s'explique notamment par le fait qu'une telle recherche présenterait un risque important pour les droits à la confidentialité et à la protection des renseignements personnels des personnes incarcérées et les exposerait à un préjudice par ailleurs évitable, «ce qui est injustifiable lorsqu'il existe d'autres mesures de prévenir la transmission du VIH» (Ibid.).

Les Directives du Commissaire du Service correctionnel du Canada (SCC) affirment expressément que les renseignements médicaux sur les détenus sont confidentiels ${ }^{9}$. Voici le texte des directives pertinentes :

8. Lord Wilberforce dans Raymond v. Honey, (1982), cité dans SHAW, p. 40; voir également les Principes fondamentaux relatifs au traitement des détenus de 1990 , Nations Unies, Annexe à la résolution 45/111 de l'Assemblée générale du 14 décembre 1990, par. 5.

9. Ces directives sont conformes aux Directives de l'OMS sur l'infection à VIH et le sida dans les prisons, OMS, Genève, mars 1993, WHO/GPA/DIR/93.3. Voici le texte des dispositions pertinentes :

31. Les informations concernant l'état de santé et le traitement médical des détenus sont confidentielles et devraient être consignées dans des dossiers uniquement accessibles au personnel de santé. Ce personnel peut donner aux directeurs des prisons ou aux autorités jưđiciaires des informations qui les aideront à traiter et soigner le patient, si celui-ci y consent.

32. Les informations concernant la séropositivité d'un individu ne peuvent être divulguées au directeur de la prison que si le personncl de santé estime, compte dûment tenu des règles de l'éthique médicale, que cela est justifié pour assurer la sécurité et lc bien-être des détenus et du personnel pénitenliaire, en appliquant dans cette đivulgation les mêmes principes que ceux qui sont généralement appliqués à la communauté dans son ensemble. Les principes et procédures concernant la notification volontaire du partenaire dans la communauté devraient être également suivis dans le cas des détenus. 33. Le stalut sérologique des détenus ne devrait jamais donner lieu à une communication de routine à l'administration pénitentiaire. Les dossiers, les cellules ou les papiers concernant les détenus ne devraient jamais comporter de signe visible (marque, étiquette. lampon, etc.) qui puissc indiquer leur état de séropositivité. 
Les détenus ont le même droit que le grand public à la confïdentialité des renseignements obtenus par un professionnel de la santé. [DC 835.12]

Tous les diagnostics d'infection à VIH doivent être inscrits dans la partie "Problèmes» des dossiers médicaux. Une fois averti, le personnel des services de santé se conformera à la Directive du commissaire $\mathrm{n}^{\circ} 835$ qui régit le caractère confidentiel et la communication de toute information obtenue par un professionnel de la santé. [DC 821.19]

Lorsque le détenu est mis en liberté conditionnelle, le personnel des services de santé doit. avec le consentement du détenu, s’assurer que des dispositions sont prises atin que le patient continue d'être suivi par un médecin qualifié. [DC 821.20]

L'état d'un détenu atteint d'infection à VIH est considéré confidentiel. Cette information ne doit pas être divulguée au personnel de surveillance ou d'organismes externes sans le consentement du détenu. [DC 821.21]

\section{I1. Exceptions à l'obligation de confidentialité}

\section{Le droit}

Dans certains cas, les renseignements médicaux confidentiels peuvent être divulgués sans qu'il y ait violation de l'obligation de confidentialité : de plus, certaines exceptions permettent ou même exigent le manquement à cette obligation. Un médecin peut divulguer des renseignements sur un patient avec le consentement éclairé de ce dernier (il ne s'agit pas alors d'une violation) ou si la loi l'exige. Toutes les provinces canadiennes et les territoires ont adopté une loi obligeant les médecins à signaler les cas de sida et, parfois, les cas d'infection à VIH, de façon nominative ou non-nominative aux représentants de la santé publique (Casswell. 1989, pp. 256257). Cependant, aucune loi particulière sur le VIH/sida n'exige ou n'autorise la divulgation, par un médecin, de ces renseignements à un partenaire sexuel ou à une personne avec qui le patient partage des seringues. Toutefois. certains territoires et provinces ont adopté une loi exigeant ou autorisant la communication, par un médecin, de renseignements sur un patient si une telle communication est nécessaire à la protection d'un tiers. La notification des partenaires est exigée par les lois sur la santé publique du Yukon et des Territoires du Nord-Ouest et autorisée, sans être exigée. par les lois sur la santé publique de certaines provinces (ibid. pp. 231-235).

En l'absence du consentement éclairé du patient et d'une loi exigeant ou autorisant la communication, la communication de renseignements sur les patients peut encore être justifiée par les doctrines du droit général. qui prévoient des exceptions à l'obligation de confidentialité. En premier lieu, la défense de nécessité peut s’appliquer afin de justifier une violation de la confidentialité lorsque «le préjudice résultant de la violation est compensé 
de toute évidence par le préjudice qu'elle permet d'éviter; le préjudice évité ne peut l'être d'une autre façon moins envahissante et est suffisamment grave pour justifier une violation de la confidentialité " (Gilmore et Somerville, 1989, p. 21 ; Somerville, 1989. pp. $211-212$; c'est nous qui traduisons). En deuxième lieu, dans de rares cas, il y a «obligation d'avertir» ou «obligation de protéger» autrui. En général, une telle obligation $s$ 'applique «seulement à des personnes identitiées ou facilement identifiables. par exemple celles qu'une personne infectée par le VIH met clairement en danger et qui n'ont aucun autre moyen de connaître ce risque, et donc de l'éviter. ou qui, à cause de leur ignorance, pourraient mettre d'autres personnes en danger» (Gilmore et Somerville. 1989, p. 21).

Dans l'arrêt Mclnerney, la Cour suprême du Canada a statué que le droit du patient à exiger que les secrets que le médecin apprend dans le cadre de sa pratique ne soient pas divulgués est absolu sauf's'il y a une raison primordiale d'y déroger :

Il peut se présenter des cas où des motifs liés à la sécurité des personnes ou du public $[\ldots]$ seraient suffisamment convaincants pour supplanter ou limiter les obligations de prime abord imposées par la relation confidentielle. (McInemey, 154, citant Halls v. Mitchell. [1928] R.C.S. 125, 136; c'est nous qui traduisons.)

Dans un autre arrêt, la Cour suprême a traité de la communication de renseignements médicaux confidentiels au cours d'une procédure judiciaire. Toutefois, elle s'est également prononcée sur la communication dans un contexte extrajudiciaire ${ }^{10}$. Elle a statué que, dans un contexte extrajudiciaire. le respect de la vie privée du particulier constitue le principe majeur qui sous-tend l'obligation d'un professionnel ou d'un hôpital de garder secrets leurs dossiers médicaux :

Un tribunal est donc en droit, dans ces circonstances, d'interpréter d'une façon libérale l'obligation générale de non-divulgation imposée aux hôpitaux et aux professionnels de la santé, et d'une façon stricte toute violation du droit à la confìdentialité (Frenette c. Métropolituine (Lu), cie d'ussurances-vie, |1992| I R.C.S. 647-675.)

Aux États-Unis, les tribunaux ont toujours déclaré que. «dans certains cas, la nécessité d'assurer la sécurité du public l'emporte sur le devoir de

10. If laut distinguer le contexte extrajudiciaire du contexte judiciaire. Dans ce dernier, il s’agil de déterminer si les communicalions entre un patient et son médecin bénéticient du privilège lorsque ec dernier est demandé au cours d'une procédure judiciaire. 
non-divulgation du médecin ${ }^{11}$ ». Dans certains États, les tribunaux ont tenu responsables envers les membres de la famille d'un patient. des médecins qui n'avaient pas dit que le patient avait une maladie contagieuse. Belitzky et Solomon ont conclu que «le médecin connaissant un diagnostic de sida qui ne communique pas ces renseignements à une victime prévisible pourrait être tenu responsable» $(1987$, p. 203 ; c'est nous qui traduisons). Dans la décision Tarasoff $v$. Regents of the University of California (17 Cal. $3 \mathrm{~d}$ 425, 551 P. 2d 334, 131 Cal. Rptr. 14 (1976)), la Cour suprême de la Californie a imposé aux psychothérapeutes l'obligation de protéger les tiers contre les actes potentiellement dangereux de leurs patients. Elle a statué que, lorsqu'un thérapeute décide ou aurait dû décider «qu'un patient présente un grave risque de violence pour autrui, il acquiert l'obligation d'exercer une diligence raisonnable afin de protéger la victime éventuelle contre un tel danger» (Belitzky et Solomon. 1987, pp. 203-204, référence a Tarasoff: c'est nous qui traduisons). Elle a conclu que «l'intérêt public, qui favorise la protection de la confidentialité des communications entre le patient et son médecin doit céder le pas dans la mesure où une telle divulgation est essentielle pour éviter des dangers à autrui».

C'est dans l'affaire Tarasoff que les tribunaux ont imposé pour la première fois l'obligation d'avertir les victimes potentielles du préjudice qui pourrait résulter des actes volontaires d'un patient. En outre, l'arrêt a confirmé qu'un médecin peut avoir l'obligation de protéger les tiers identifiés contre les dangers résultant de la maladie d'un patient ${ }^{12}$.

\section{Les obligations éthiques}

L'obligation éthique d'assurer la confidentialité des renseignements communiqués au médecin par son patient n'est pas absolue. Au cours de son assemblée annuelle de 1987, l'Association médicale canadienne a décidé qu'ils n'est pas, pour un médecin, contraire à l'éthique de «communiquer de façon discrète. à une personne appropriée, au su du patient», mais sans son consentement, le fait que le patient est infecté par le VIH «lorsque l'intérêt public [dans une telle divulgation] l'emporte de toute évidence sur

11. Belitzky ct Solomon (1987), p. 203, note 24, référence à $E d w a r d s ~ v . ~ L a m b, 69$ N.H. 599, 45 A.480 (1899); Earle v. Kuklo, 26 N.J. Super. 471, 98 A.2d 107 (1953) (le propriétaire expose les locataires à la tuberculose); Kliegel v. Aitken, 94 Wis. 432, 60 N.W.67 (1896) (l'employeur expose les employés à la fièvre typhoïle); Davis v. Rodman, 147 Ark. 385, 227 S.W. 612 (1921) (fièvre typhö̈de); Skillings v. Allen, 143 Minn, 323, 173 N.W. 663 (1919) (scarlatine): Edwards, 69 N.H. 599, 45 A.480 (blessures inlecticuses); Fosgate v. Corona, 66 N.J. 268, 330 A.2d 355 (1974) (tuberculose); Wojick v. Aluminitum Company of America, 18 Misc. 2d 740, 183 N.Y.S.2d 351 (Sup. Ct. 1959) (l'employeur fait passer des radiographies de façon régulière à un cmployé responsable de ne pas avoir divulgué sa tuberculose).

12. Pour une autre discussion de l'affaire Tarasoff et d'autres décisichis américaines, voir Belitzky et Solomon (1987), pp. 204-207. 
l'intérêt du patient» (Association médicale canadienne (1987) 137 J.A.M.C. 653; cité dans Casswell, 1989, pp. 226-227. notes 4 et 5 ; c'est nous qui traduisons). Dans son exposé de position sur le $\mathrm{VIH} /$ sida publié en 1989. 1'Association a souligné la nécessité de respecter la confidentialité des patients infectés par le VIH et elle a recommandé l'adoption de garanties juridiques et réglementaires afin de protéger cette confidentialité. Toutefois, elle avisait également les médecins du fait qu'il n'est pas nécessairement contraire à la déontologie médicale et, en fait, qu'il peut être approprié de révéler l'état de leur patient atteint à son conjoint ou à son partenaire sexuel «lorsque le patient refuse de le faire lui-même» (Association médicale canadienne. 1989,64C). Elle poursuivait en disant qu'«[u]ne telle révélation serait acceptable du point de vue déontologique si toutes les conditions suivantes sont réunies : il y a lieu de croire que le conjoint ou le partenaire sexuel du patient atteint n'a pas de raison de croire qu'il est exposé à une infection par le VIH; le patient atteint a refusé d'informer son conjoint ou son partenaire sexuel de son état; le patient atteint a refusé l'offre du medecin de le faire en son nom; et le médecin a informé son patient de son intention de le faire» (ibid.).

De la même façon, l'American Medical Association a fait observer que «la confidentialité de la relation médecin-patient est d'une importance vitale, mais non absolue» (Dickens, 1990, p. 98, référence à l'American Medical Association. 1987; c'est nous qui traduisons). Dans son rapport provisoire sur la politique relative au sida, l'Association proposait le principe fondamental suivant, à savoir que «l'accès aux renseignements sur les patients devrait se limiter au personnel soignant qui en a besoin pour aider le patient ou protéger la santé des autres qui lui sont liés de près» (American Medical Association, 1987, p. 2102 ; c'est nous qui traduisons). Dans le rapport, elle reconnaissait que. dans certaines circonstances limitées, il peut être nécessaire d'avertir les tiers :

Les médecins qui ont des raisons de croire qu'une personne infectée a un partenaire sexuel qui ne se doute de rien devraient être incités à informer les autorités de la santé publique. L'obligation d'avertir le partenaire sexuel qui ne se doute de rien devrait alors incomber aux autorités de la santé publique qu'à la personne infectée, et non pas au médecin de la personne infectée. (lbid., p. 2103.)

\section{La politique carcérale}

Les Directives du Commissaire du SCC reconnaissent de façon explicite que l'obligation de confidentialité des renseignements médicaux sur les détenus n'est pas absolue. Dans les établissements correctionnels comme à l'extérieur, certaines personnes peuvent exposer autrui au VIH, notamment par l'usage de la force. la coercition ou la contrainte. La communication de renseignements médicaux sur les personnes susceptibles d'exposer autrui 
au VIH de façon non consensuelle ou par la force peut à l'occasion être justifiée (Somerville et Gilmore, 1988, p. 53).

Voici le texte des Directives:

[S]'il y a raison de croire qu'un détenu représente un danger pour sa sécurité ou celle d'autres personnes. il incombe au professionnel de la santé. tout en se conformant à la Loi sur la protection des renseignements personnels. de communiquer cette information au personnel compétent sans le consentement du détenu. [DC 821.21]

Sur la question de la divulgation de renseignements médicaux relatifs aux détenus, le Commissaire à la protection de la vie privée du Canada a déclaré qu' "[o]n peut toutefois reconnaître un certain fondement à une politique qui autorise la divulgation lorsque la conduite du détenu constitue une menace pour autrui - qu'ils s'agisse de pratiques sexuelles à risque ou de l'utilisation de seringues en commun " (Le Commissaire à la protection de la vie privée, 1989, p. 47). Il a fait une distinction entre la divulgation de renseignements médicaux sur un détenu dans le but d'éviter l'exposition d'autres détenus à l'infection à VIH et la divulgation ayant pour objet d'éviter l'exposition du personnel correctionnel à l'infection à VIH. Dans son rapport, il souligne que la divulgation liée au personnel correctionnel «reste à déterminer» (ibid., p. 47). Cette attitude reflète en partie la préoccupation du Commissaire à la protection de la vie privée, qui craint que les agents de correction ne transmettent, par négligence. ces renseignements à d'autres détenus. Le Commissaire reconnaît que, «étant donné les relations hostiles entre les détenus et les agents, ces derniers risquent parfois de craindre que les détenus cherchent à les contaminer». Toutefois, il conclut qu' «[i]l y a lieu d'évaluer la gravité de ce risque avant de décider s'il faut informer les agents des Services correctionnels qu'un détenu est porteur du VIH».

Récemment. le Comité consultatif national sur le sida (CCN-sida) a recommandé que dans les établissements correctionnels. tous les renseignements relatifs au VIH soient tenus strictement confidentiels, et ce pour deux raisons. D'une part, le CCN-sida se préoccupait de ce que, si la confidentialité de l'information était violée, les détenus infectés risqueraient fort de faire l'objet de discrimination et devraient en toute probabilité être mis à l'écart de la population carcérale. D'autre part, il estime qu' «aucune raison ne justifie la divulgation de tels renseignements soit aux autres détenus, soit au personnel non médical de l'établissement pénitentiaire». Par conséquent. le $\mathrm{CCN}$-sida a recommandé que le solliciteur gênéral adopte des mesures supplémentaires pour garantir la confidentialité de tous les renseignements sur le VIH dans les établissements correctionnels. Plus particulièrement, il a jugé nécessaire «de prévoir des mesures disciplinaice à l'encontre de tout employé qui dérogerait à ces règles» (Comité consultatif national sur le sida, 1992, p. 17). 


\section{Résumé}

Selon la présomption absolue régissant la confidentialité des renseignements médicaux. notamment dans les cas de sida, tous, y compris les détenus, ont droit au respect de leurs droits. à la protection des renseignements personnels et à la confidentialité, droits qui ne peuvent être violés que pour des raisons graves et sur présentation d'une justification adéquate (Somerville, 1989, p. 211).

La divulgation ne peut être justifiée des points de vue du droit et de l'éthique en l'absence d'un préjudice potentiel véritable pour un tiers identifiable. Ainsi que l'a déclaré le Groupe de travail fédéral-provincialterritorial sur la confidentialité concernant la séropositivité a l'égard du VIH, «les renseignements sur l'état sérologique ne devraient être divulgués qu'au profit de la personne ayant subi le test. par exemple lorsque c'est nécessaire pour assurer des soins médicaux appropriés ou qu'il est raisonnable de croire que la conduite d'une personne infectée par le VIH constitue un risque inacceptable pour la santé d'autrui» (Groupe de travail fédéral-provincial-territorial sur la confidentialité concernant la séroposivité à l'égard du VIH, 1988).

Sur le plan juridique, la divulgation sans le consentement éclairé n'est justifiée que s'il peut être prouvé qu'on ne dispose d'aucun autre moyen moins envahissant et moins intrusif de protéger autrui et que la divulgation est nécessaire à l'atteinte de cet objectif et susceptible d'être efficace.

Les analyses éthique et juridique concordent sur ce point (Somerville et Gilmore, 1988, pp. 13-14). L'analyse éthique implique l'évaluation des avantages, des risques et des préjudices pour le détenu au sujet duquel les renseignements seraient divulgués, pour la personne à qui les renseignements seraient divulgués et pour la société. Par conséquent. une telle divulgation serait justifiée, d'un point de vue éthique, si les conditions minimales suivantes étaient respectées (Gilmore. Somerville, Jürgens et Wilson, 1993) : 1) les préjudices à éviter doivent être graves et probables et ne sont pas évitables sans la divulgation: 2) les personnes qui subiront le préjudice doivent être identifiables, c'est-à-dire que la crainte générale d'un préjudice pour une population, comme les prisonniers ou les gardiens. serait trop vague pour justifier la divulgation : 3 ) les avantages découlant de la divulgation doivent l'emporter sur les risques et les préjudices qu'elle entraînerait pour la société et le détenu; 4) la divulgation doit être le moyen le moins envahissant et le moins nuisible, susceptible d'être efficace, qui permette d'atteindre l'objectif en vue duquel la confidentialité serait violée.

En outre, la justification de l'exception incombe à celui qui invoque cette dernière, et non pas au détenu qui bénéficie du droit à la confidentialité (Gilmore et Somerville, 1989. p. 6). De plus, l'exception ne s'applique que lorsque le détenu n'a pas consenti à la divulgation (ou a refusé de 
l'autoriser), ce qui sous-entend que le détenu doit être informé, préalablement. que des renseignements médicaux le concernant seront divulgués sans son consentement.

\section{LE «BESOIN DE SAVOIR » OU LA NÉCESSITÉ DE LA DIVULGATION}

Quand on parle de la nécessité de la divulgation de renseignements médicaux confidentiels sur les détenus, on utilise souvent l'expression "besoin de savoir». L'analyse qui suit porte sur ce «besoin». Les auteurs examinent d'abord l'objectif visé par la divulgation (le but pour lequel elle serait nécessaire). Ils analysent ensuite la nécessité d'une telle divulgation - en particulier si elle permettrait d'atteindre cet objectif - et les solutions de rechange, y compris l'analyse des avantages, des risques et des préjudices résultant de la divulgation. Enfin, ils examinent des cas précis pouvant donner lieu à une demande de divulgation de renseignements.

\section{L'objectif de la divulgation}

La communication de renseignements médicaux sur un détenu peut être demandée dans deux cas : avant l'exposition au VIH, afin d'en éviter la transmission, et après l'exposition au VIH. Ces deux situations sont discutées sé parément.

\section{Divulgation avant l'exposition au VIH}

Dans ce cas, l'objectif de la divulgation est d'éviter ou de réduire au minimum l'exposition au VIH. La divulgation donnerait lieu à une intervention destinée à empêcher l'exposition.

Dans les pénitenciers fédéraux, les employés ont souvent prêtendu qu'ils avaient besoin de connaître l'état sérologique des détenus infectés afin de prendre les précautions nécessaires pour se protéger eux-mêmes ainsi que leurs familles respectives. Sur 450 membres du personnel, 367 (soit $81,6 \%$ ) ont répondu à cette question dans le cadre d'un questionnaire qui leur avait été envoyé par le CESP en disant qu'ils avaient besoin de connaître les résultats des tests de VIH des détenus ${ }^{13}$. En particulier,

13. Le conité a envoyé à des comités de détenus des établissements fédéraux un questionnaire dans lequel les détenus devaient exprimer leurs opinions ct leurs préoccupations sur l'éducation liéc au VIH/sida et à la consommation de drogue, sur la possibilité de se procurer des condoms, des aiguilles et des seringues propres ou encore du matériel pour les nettoyer et, enfin, sur les tests el la confidentialité. Le comité les invitait aussi à lui transmettre lcurs propositions, leurs recommandations ou leurs commentaires sur le VlH/sida c1 la consommation de drogue dans les prisons. Le comité a envoyé un deuxième questionnaire, aux employés travaillant dans les prisons, pour recueillir des renseignements sur leurs prénccupations à l'égard de la sécurité et sur celles des autorités carcérales a l'égard du maintien de la sécurité et de l'ordre dans les établissements. 
certains ont soutenu qu'il n'était pas pratique de recourir à des précautions universelles et qu'il est irréaliste de croire que des précautions pouvant empêcher la transmission du VIH peuvent être appliquées universellement dans les pénitenciers. Ils ont aussi allégué qu'ils seraient mieux protégés s'il savaient quels détenus sont infectés par le VIH, puisqu'ils pourraient alors prendre des précautions accrues avec eux.

\section{Divulgation après l'exposition au VIH}

Dans ce cas, l’objectif de la divulgation est d'éviter ou de réduire au minimum le risque d'infection par le VIH ou de rassurer la personne qui a pu y être exposée. Des employés ont prétendu que le personnel soignant devrait pouvoir communiquer des renseignements médicaux à un membre du personnel ou à un détenu qui a été exposé aux liquides organiques ou au sang d'un détenu porteur du VIH ou de l'hépatite B. La divulgation entrâ̂nera une intervention destinée à empêcher l'infection après l'exposition ou. à tout le moins, à rassurer la personne exposée. Quant à l'exposition au virus de l'hépatite $B$, un traitement à la gammaglobuline et un vaccin contre l'hépatite B seraient administrés ; pour ce qui est de l'exposition au $\mathrm{VlH}$, il s'agirait d'un traitement à la zidovudine (AZT).

\section{La divulgation est-elle nécessaire?}

Dans cette section, les auteurs examinent la nécessité de la communication - à savoir, en particulier si la divulgation permettrait d'atteindre les objectifs susmentionnés - et les solutions de rechange. Afin d'établir si la divulgation est nécessaire, il est important de bien comprendre les faits scientifiques concernant le VIH/sida.

\section{Les modes de transmission du VIH}

Une personne peut être exposée au VIH dans trois cas : premièrement, elle peut être exposée aux fluides corporels - autres que le sang ou les liquides sanguins - d'un individu intecté par le VIH; deuxièmement, elle peut être exposée à du sang ou à des liquides sanguins infectés par le VIH; troisièmement. elle peut être exposée au sperme. aux sécrétions vaginales ou au sang lors d'une relation sexuelle avec une personne infectée. Les probabilités d'infection par le VIH diffèrent pour chacun de ces trois cas. L'exposition au VIH n'entraîne pas nécessairement une infection au VIH. C'est dire que l'infection est possible, mais non pas que la probabilité va se concrétiser. Le risque ou la probabilité de la transmission du VIH varie beaucoup. Ils dépendent du type d'exposition (contact de la peau ou des muqueuses, ou inoculation par la peau), du liquide organique (sang, sperme ou sécrétions vaginales), du volume du fluide porteur en cause dans l'exposition ainsi que de la concentration de VIH dans le fluide. Pour certaines expositions, ce risque est jugé négligeable. 
Le simple contact physique avec une personne infectée par le VIH et le simple contact de la peau avec des fluides corporels infectés par le VIH, par exemple la transpiration, les larmes, l'urine ou les matières fécales. n'ont jamais été associés à des cas documentés de transmission du VIH (Lif́son. 1988: Friedland, Kahl et coll., 1990; Gershon. Vlahov et coll., 1990; Rogers et Gellin. 1990). Il n'est pas nécessaire d'appliquer des précautions universelles lorsque le risque est négligeable. Cependant, le recours à celles-ci dans de tels cas éliminera la possibilité d'une exposition au VIH et à d'autres agents infectieux.

Certaines personnes exposées à du sang ou à des fluides sanguins infectés par le VIH ont été infectées par le VIH. Toutefois, cela ne s'est produit que rarement et uniquement lorsque le sang avait été introduit à l'intérieur du corps. En milieu de soins de santé, l'exposition survient le plus souvent lorsqu'un pourvoyeur de soins est blessé et que du sang s'introduit dans son corps par une blessure, par exemple lorsqu'il est piqué par une aiguille souillée de sang ou un autre instrument creux. Par ailleurs, les aiguilles pleines (comme les aiguilles à suturer) n'ont pas été associées à des cas documentés de transmission du VIH. La probabilité d'une infection à VIH après des blessures accidentelles avec de telles aiguilles a été quantifiée dans des études prospectives (Mikes et coll., 1991 ; cité dans AIDS Reference Guide, Washington, AIDS Information Service, Inc., janvier 1992, 907.1-907.18). Un cas d'infection a été documenté pour chaque groupe de 333 blessures. Ce chiffre peut être une surestimation, parce que de nombreux accidents donnant lieu à une exposition ne sont pas signalés. Quant au milieu carcéral, un seul cas documenté de transmission du VIH à un employé du milieu a été signalé. Il s'agit d'un agent qui avait été poignardé au moyen d'une seringue contenant du sang infecté par le VIH ${ }^{14}$. On pense que trois pourvoyeurs de soins ont été infectés après avoir été éclaboussés avec du sang infecté par le VIH, mais ce cas n'est pas prouvé (Centers for Disease Control, 1987). On ne sait pas de façon précise comment le virus s'est introduit dans leur corps, mais ils avaient peut-être déjà la peau endommagée. ce qui les prédisposait à une infection à VIH. Les morsures et les égratignures n'ont pas été associées de façon convaincante à la transmission du VIH (Tsoukas et coll., 1988; Richman et Rickman, 1993). Enfín. on a signalé un seul cas de transmission du VIH à un joueur de soccer qui s'était heurté à un joueur infecté par le VIH, les deux joueurs ayant subi de graves lacérations (Torre et coll., 1990).

14. Ce cas s'est produit en Australic. On a allégué qu'un jeune agent, nouvelle recrue dans le service, a êté attaqué et poignardé avec une seringue remplie de sang. Par la suite, l'agent a cu un résultat positif pour le VIH. On a beaucoup parté de cel incident dans les journaux. Voir Doyle (1991). 
Une personne infectée peut transmettre le VIH à une autre personne avec laquelle elle partage des aiguilles et des seringues contaminées par son sang infecté et par des relations sexuelles non protégées. Encore une fois. ces activités exposeront au VIH la personne non infectée, mais celle-ci ne sera pas nécessairement infectée. Le fait de ne pas avoir de relations sexuelles ou de ne pas partager des seringues pour consommer des drogues ou pour se faire tatouer éliminera le risque d'infection, alors que l'utilisation du condom ou le nettoyage des seringues avant chaque utilisation réduiront ce risque.

\section{Les précautions nécessaires}

Il existe principalement deux approches pour prévenir l'exposition au VIH. La première est de ne pas avoir d'activités donnant lieu à une exposition, par exemple des activités sexuelles non protégées et l'utilisation de seringues contaminées, et d'éviter le contact physique avec un détenu violent. La deuxième est de réduire le risque de transmission du VIH lors des relations sexuelles, de la consommation de drogues injectables ou du tatouage, lors d'un contact avec un détenu violent et chaque fois qu'une blessure donnant lieu à une exposition peut se produire (par exemple au cours de la fouille des cellules).

Dans chacune de ces situations, le fait de savoir qu'une personne présente est infectée ne fera que confirmer la nécessité de prendre des précautions afin de réduire ou d'éliminer le risque d'exposition. Lorsqu'on n'est pas certain qu'une personne n'est pas infectée, les mêmes précautions s'appliquent. Les précautions pourraient être assouplies seulement si on était certain que les personnes en cause dans une situation où l'exposition est possible ne sont pas infectées. Comme on ne peut jamais en être certain, il faut prendre des précautions avec quiconque est en cause dans des situations donnant lieu à une exposition, c'est-à-dire qu'il faut appliquer des précautions universelles. Les auteurs analysent différentes situations survenant dans les établissements correctionnels au cours desquelles des personnes peuvent être exposées au VIH.

\section{(1) L'exposition au sang ou aux fluides sanguins}

Dans tous les cas où le personnel ou les détenus sont exposés à du sang ou à des fluides sanguins, ils doivent prendre des précautions universelles pour éviter l'exposition au VIH et l'infection, peu importe que le personnel, les directeurs ou les détenus sachent ou non si un détenu ou un membre du personnel est ou n'est pas infecté par le VIH ${ }^{15}$. Cette recommandation est conforme aux lignes directrices de lutte contre l'infection. élaborées pour le

15. Pour une description de ces précautions dans le secteur de la santé, voir Santé el Bien-êtrc social Canada (1987). 
personnel du SCC en 198916. Puisqu'il est impossible de détecter ou d'identifier tous les détenus infectés par le VIH, le personnel ne serait pas protégé contre la transmission $\mathrm{du}$ VIH si des mesures de précaution n'étaient prises qu'avec les détenus que l'on sait infectés. Il est impossible d'identifier tous les détenus infectés pour deux raisons. Premièrement, tous les détenus ne subissent pas un test de dépistage ${ }^{17}$. La crainte de la divulgation ainsi que les risques et les préjudices pour le détenu, si les renseignements touchant son statut sérologique sont connus, constituent un fort facteur de dissuasion à l'encontre du dépistage pour de nombreux détenus. Un manque de confiance apparent dans le personnel médical de l'établissement est un autre élément qui dissuade le détenu de subir un test. En l'absence de garanties fermes de non-divulgation, seul les tests anonymes assureraient la confidentialité des renseignements médicaux. Cependant, de tels tests sont rarement offerts aux détenus, sinon jamais, et c'est habituellement le personnel médical de l'établissement qui fait passer les tests. Deuxièmement, les tests de dépistage des anticorps anti-VIH ne permettront pas de détecter tous les détenus infectés. Certains auront été infectés trop récemment pour pouvoir être détectés, et d'autres, quoique rarement, obtiendront de faux résultats négatifs. Il peut s'écouler jusqu'à six mois entre le moment de l'infection par le VIH et la détection de l'infection, ce qui veut dire qu'un résultat négatif ne peut fournir de renseignements "utiles" pour ce qui est de la protection et que l'on ne peut se permettre de relâchement à cet égard. En conséquence, il faut appliquer de façon soutenue et universelle les précautions qui peuvent prévenir la transmission du VIH. La divulgation au personnel du statut sérologique des détenus que l'on sait atteints enverrait le mauvais message : que le personnel doit se protéger

16. Lignes directrices intérimaires de lutte contre l'infection du personnel. Service correctionnel du Canada, Ottawa, Approvisionnements et Services Canada, 1989. Ces lignes directrices contiennent des renseignements sur les précautions générales que tout le personnel doit suivre afin d'empêcher la propagation de l'infection, ainsi que les procédures que doit suivre l'êtablissement touchant le nettoyage et la désinfection du milieu, de l'equipernent et des foumitures, le nettoyage des cellules, des cuisines et de la buanderie, ainsi que «les précautions à prendre avec les détenus identifiés conme ayant une maladic inlectieuse». Ces lignes directrices soulignent qu'il faut appliquer des précautions universelles avec le sang et les lluides corporels, «qu'il y ait ou non un risque identilié », el que "la sécurité du personnel est mieux assurée si l'on suppose que tout individu peut être infecte d'une maladic transmissible."

17. Conformément à la Directive du Commissaire $n^{\circ} 821$, le test de dépistage des anticorps anti-VIH, dans les établissements lédéraux, est volontaire, c'est-à-dire qu'il se fait avec le consentement éclairé du détenu. Cette recommandation est conforme au principe général régissant le test de dépistage des anticorps anti-VIH au Canada, sclon lequel ce test est administré sur une base volontaire. c'est-à-dire qu'il se láit avec le consentement échairé de l'intéressé, si des séances de counselling el des services d'éducation avant et après le test sont ollertes, el si la contidentialité des résultats ou l'anonymal du test peuvent être assurés. Voir Sonerville et Gilmore (1988), p. xiii. 
seulement lorsqu'il sait qu'un détenu est infecté, plutôt que d'appliquer des précautions universelles, et que le personnel peut prendre des précautions additionnelles avec les détenus qu'il sait infectés. plutôt que de toujours prendre des précautions suffisantes. Il faudrait plutôt éduquer le personnel et l'inciter à toujours prendre des précautions. Ceux qui prétendent que des précautions supplémentaires pourraient être prises avec les détenus dont la séropositivité est connue sont mal avisés, puisque la notion de précautions universelles suppose l'adoption des mêmes précautions pour tous les détenus, que la séropositivité soit connue ou non.

Les membres du personnel ont parfois dit qu'il était impossible ou irréalisable d'appliquer des précautions universelles en milieu carcéral. Toutefois, l'expérience en milieu de soins de santé a prouvé qu'on peut et qu'on doit apprendre à user de précautions universelles. Par exemple, dans les hôpitaux, où le rísque d'exposition au VIH est beaucoup plus élevé que dans les prisons, on a depuis longtemps compris que la divulgation de l'état sérologique des patients pour le VIH n'accrô̂trait pas la sécurité du personnel (Association médicale canadienne, 1993 ; Laboratoire de lutte contre la maladie. 1992, p. 178; Gerberding et coll., 1990). Aussi l'état sérologique des patients et des médecins n'est-il pas révélé, et l'on adhère rigoureusement à des précautions universelles. On risquerait de créer un faux sentiment de sécurité en informant systématiquement le personnel de l'état sérologique des détenus infectés par le VIH. Puisque tous les prisonniers ne sont pas soumis à des tests de façon régulière, le personnel ne connaîtrait la séropositivité que de quelques prisonniers. On craint en outre que si la sćropositivité des détenus était divulguée au personnel, moins de détenus demanderaient à être soumis à des tests ou déclareraient eux-mêmes leur séropositivité pour le VIH. ce qui réduirait le nombre de détenus dont la séropositivité est connue. Selon l'expérience acquise auprès des détenus séropositifs, la plupart de ces derniers déclarent tôt ou tard leur état à leurs codétenus et au personnel. Une telle divulgation est volontaire, et ils attendent d'être prêts à le dire.

Comme Casswell le déclarait, «i! peut être plus facile d’assurer la protection du personnel en l'informant de la nécessité de se protéger luimême [c'est-ì-dire avoir recours à des précautions universelles] et en lui inculquant le sens des responsabilités plutôt que par des avertissements. Ces derniers ne sont pas forcément efficaces, puisqu'ils peuvent être donnés au mauvais moment, ne pas se rendre jusqu'aux partenaires fles détenus infectés! ou être fondés sur de faux résultats aux tests" (Casswell, 1989. p. 248 ; c'est nous qui traduisons).

(2) La violence

Les précautions universelles élimineront le risque de transmission du VIH provenant des éclaboussures de sang, mais il est essentiel de prévenir 
les blessures donnant lieu à une exposition afïn de réduire ou d'éliminer le risque beaucoup plus grand de transmission du VIH dans les cas de blessure. Ce principe s'applique tant en milieu de soins de santé qu'en milieu carcéral. La réduction de l'incidence des blessures permettra de réduire l'incidence de l'exposition au VIH, ce qui veut dire, en milieu carcéral, prévenir les accidents, par exemple les blessures accidentelles avec des aiguilles au cours de la fouille des cellules, et prévenir la violence qui entraine des blessures et une exposition au sang. Il faudrait donc porter une attention méticuleuse aux pratiques de sécurité (pratiques de fouille plus sécuritaires), utiliser des gants résistants à la perforation, au besoin, et maîtriser les détenus agressifs, en particulier éviter ou réduire les contacts physiques avec eux lorsqu'ils ont un comportement violent.

En ce qui concerne la maîtrise des détenus agressifs. certains prétendent que. dans plusieurs cas. «les précautions prévues ne parviennent pas à écarter le risque» (Zazulak. 1992: c'est nous qui traduisons). Mentionnons, par exemple, «les incidents au cours desquels un détenu violent qui saigne doit être maîtrisé par un membre de notre [SCC] personnel » (ibid.). Dans presque tous ces cas, si ce n'est la totalité d'entre eux, l'absence de contacts physiques entre le personnel et le détenu violent protégera le personnel en éliminant les blessures qu'il pourrait subir. L'élimination des blessures du personnel et le recours approprié à des précautions universelles élimineront par conséquent le risque de transmission du VIH par une exposition au sang du détenu. De nouveau, et pour les raisons mentionnées ci-dessus, toute mesure ou procédure prévue afin de prévenir ou de réduire l'exposition au VIH doit être (ou devrait être) appliquée, que l'on sache ou non si le détenu dont la cellule est fouillée ou qui fait preuve d'agressivité est infecté par le VIH.

\section{(3) L’activité sexuelle consensuelle}

Il n'existe aucune donnée fiable sur la prévalence de l'activité sexuelle consensuelle dans les prisons canadiennes. Cependant. il y a lieu de croire que ce genre d'activités a cours et qu'il est peut-être répandu. La fréquence des rapports sexuels varie selon plusieurs facteurs, par exemple selon que les détenus sont logés dans une cellule individuelle ou un dortoir, selon la durée de la peine, la classification de sécurité et la fréquence autorisée des visites conjugales (Heilpern et Egger, 1989, p. 40, où il est fait état de Hough et Schwartz, 1985). Il a été avancé que l'activité homosexuelle a cours dans les prisons, comme à l'extérieur, en tant qu'expression de l'orientation sexuelle. De plus, les conditions de la vie en prison favorisent les relations homosexuelles dans l'établissement (Thomas, 1990, p. 5). Plusieurs études sur l'ampleur de l'activité sexuelle dans les prisons aux ÉtatsUnis indiquent qu'entre 10 et 30 pour cent des détenus ont des rapports sexuels (Decker et coll.: Nacci et Kane, 1982). 
Des condoms ont été distribués aux détenus dans les établissements correctionnels fédéraux à compter du $1^{\text {er }}$ janvier 1992, pour freiner la transmission du VIH. Chaque pénitencier a établi son propre système de distribution de condoms. Dans certains établissements, on distribue des condoms à tous les détenus et l'on en met une certaine quantité à leur disposition dans les unités résidentielles; ailleurs, la tâche de fournir les condoms est du ressort exclusif du personnel du service de santé de l'établissement. C'est d'ailleurs ce qui se produit dans les établissements de détention provinciaux québécois. où ce mode de distribution a été choisi puisqu'il «permet de transmettre un message éducatif en même temps». Pourtant. un nombre considérable de détenus risquent de ne pas se prévaloir de ce service, en raison du manque de confidentialité, par crainte d'être identifiés comme ayant des activités homosexuelles et de faire l'objet de discrimination.

La décision de distribuer des condoms aux détenus dans les établissements correctionnels fédéraux a été très bien accueillie et n'a posé aucun problème dans les établissements. Ceci est conforme à l'expérience d'autres systèmes carcéraux où l'on a observé, après des années d'expérience, que la distribution des condoms n'avait pas causé de problèmes de sécurité ni d'incidents fâcheux.

Il a été allégué que la distribution de condoms et l'éducation des détenus sur la transmission du VIH pouvaient ne pas être suffisantes pour parer au risque de transmission du VIH par les relations sexuelles entre les détenus, et que pour mieux protéger les détenus, on devrait communiquer aux pairs l'identité des détenus infectés. Pourtant, en général, ceci nuirait aux messages transmis touchant les pratiques sexuelles plus sécuritaires, c'est-à-dire le recours à des précautions d'application universelle. Il est inutile de savoir qui est infecté lorsque les précautions appropriées sont prises. Il n'existe qu'une exception à cette règle. Lorsqu'une personne ne sait pas que son partenaire sexuel est infecté par le VIH et qu'elle n'a aucune raison de $s^{\prime}$ en douter, il peut être nécessaire de l'informer des risques de transmission du VIH. C'est le cas, par exemple, si la personne a une relation monogame ou a long terme avec un partenaire qui refuse de révéler à son partenaire qu'elle est infectée et s'ils ont des activités par lesquelles le VIH peut se transmettre. Cette situation est analogue a la notification du partenaire à l'extérieur du milieu carcéral.

La façon la plus efficace de réduire davantage la transmission da VIH par l'activité sexuelle ne serait pas de divulguer l'état sérologique, mais de trouver des meilleures méthodes de distribution de condoms et d'encourager davantage leur utilisation. C'est pourquoi le Comité sur le sida et les prisons a recommandé que les établissements de détention prennent les mesures nécessaires pour que les détenus puissent se procurer des condoms. 
des digues dentaires et des lubrifiants à base d'eau facilement et d'une manière discrète, et non seulement au service de santé de l'établissement. Le Comité a recommandé également qu'une trousse comprenant. entre autres. des condoms, des digues dentaires et du lubrifiant soit remise à chaque détenu à son arrivée en prison. Le détenu disposerait ainsi du matériel nécessaire pour se protéger contre l'infection à VIH sans avoir à demander ou à aller chercher ce matériel et risquer ainsi d'être vu du personnel et des autres détenus. Cette mesure contribuerait également a «déstigmatiser» le phénomène de l'activité sexuelle en prison et à encourager une discussion plus franche à ce sujet.

Dans les pénitenciers fédéraux. l'activité sexuelle consensuelle entre les détenus est encore considérée ou perçue comme une activité «susceptible de mettre en danger la sécurité du pénitencier » selon l'alinéa $40 \mathrm{~m}$ ) de la Loi sur le système correctionnel et la mise en liberté sous condition. Ceux qui prétendent que l'activité sexuelle entre les détenus doit continuer d'être considérée comme une infraction disciplinaire allèguent souvent que les prisons ne sont pas des lieux privés et que, par conséquent, les actes sexuels commis en prison constituent des infractions criminelles. Elle allèguent ́́galement que la suppression de l'activité sexuelle entre les détenus de la liste des infractions disciplinaires donnerait à conclure qu'on ferme les yeux sur l'activité homosexuelle, ou même qu'on l'encourage. D'autres personnes sont conscientes de la contradiction entre l'interdiction pour les détenus d'avoir des rapports sexuels entre eux et l'accès libre à des condoms. D'autres encore estiment que l'activité sexuelle consensuelle devrait être autorisée non seulement parce que cette mesure permettrait d'accroître l'efficacité de la prévention du VIH (les détenus disent souvent craindre de se voir imposer une sanction disciplinaire s'ils étaient pris «sur le fait »; obligés d'agir furtivement par crainte d'être découverts. les détenus ne prennent pas souvent les précautions qu'exigent les pratiques sexuelles sans risque), mais également parce qu'interdire les rapports sexuels entre les détenus constitue une violation des droits de la personne. Le Comité sur le sida et les prisons a recommandé que l'activité sexuelle consensuelle soit supprimée de la liste des intractions disciplinaires. Le Comité estime que cette mesure permettrait aux détenus de prendre le temps pour utiliser les précautions nécessaires et qu'elle serait conforme aux principes directeurs généraux qui guident le SCC. Elle serait d'ailleurs appelée par les principes en question. selon lesquels «les mesures nécessaires à la protection du public, des agents et des détenus doivent être le moins restrictives possibles» et le détenu «continue à jouir des droits et privilèges reconnus à tout citoyen. sauf de ceux dont la suppression ou restriction est une conséquence nécessaire de la peine qui lui est infligée " 
(alinéas 4d) et e) de la Loi sur le sustème correctionnel et la mise en liberté sous condition ${ }^{18}$ ).

(4) L'activité sexuelle non consensuelle

Le rapport sexuel non consensuel représente un autre risque de transmission du VIH dans les prisons. Il est peu probable que. dans un tel cas, on utilise les précautions appropriées contre la transmission du VIH par voie sexuelle, et la victime ne sera pas protégée. Cependant. le fait de savoir que l'agresseur est infecté par le VIH ne protégera pas non plus la victime contre l'agression sexuelle et le risque inhérent de transmission du VIH. Il faut adopter une démarche qui, en premier lieu, permettra de prévenir ou de réduire les agressions sexuelles. Ce n'est que lorsqu'un détenu séropositif a déjà commis ou menacé de commettre une agression sexuelle que peut être justifiée la divulgation à certains employés de son infection par une maladie infectieuse, mais non pas de l'infection par le VIH, afin qu'ils puissent le surveiller de plus près.

(5) L'usage de drogues injectables

Il n'existe pas de données fiables sur la fréquence de l'usage de drogues injectables ou autres en milieu carcéral au Canada. Les seules données disponibles portent sur la fréquence de l'usage de drogues par les détenus avant leur incarcération. On utilise un questionnaire informatisé sur le mode de vie pour obtenir des renseignements sur l'étendue de la consommation de drogues et d'alcool par les détenus et la nature de ces substances. Sur les 371 détenus ayant répondu au questionnaire en 1989-1990, plus de $10 \%$ ont admis avoir consommé des drogues tous les jours dans les six mois précédant leur incarcération, $17 \%$ ont déclaré qu'ils buvaient régulièrement et $64 \%$ ont dit qu'ils avaient consommé de l'alcool ou d'autres drogues le jour de la perpétration de leur crime. En outre. plus de la moitié de la totalité des détenus sous responsabilité fédérale étaient considérés comme ayant un grave problème de toxicomanie (Riley, 1993, p. 3, où il est fait mention du Service correctionnel du Canada, 1990). Le Comité parlementaire sur le sida a entendu des témoignages selon lesquels jusqu’à $50 \%$ des détenus pourraient consommer des drogues (Comité parlementaire sur le sida, 1990, p. 47), et le Groupe d'étude sur la réduction de la

18. Il est important d'établir une nette distinction entre l'activité scxuelle consensuelle et l'activité sexuelle non consensuclle. Alors que celte dernière ne peut être toléréc et pourrait meltre en danger la sécurité du pénitencier, l'activité sexuelle consensuelle ne pourrait causer des «problèmes » qu'indirectement en ratison des réactions négatives ou d'une intolérance de la part du personnel, des autres détenus ou de la population à l'égard des détenus qui ont des rapports sexucls. Cependant, ces réactions découlent des préjugés qu'entretiennent ces personnes plutôt qu'elles ne s'expliquent par l'activité sexuelle en soi, de sorte qu'il y aurait lieu de discutcr plus librement de la question de la sexualité. 
toxicomanie de 1991 a reconnu que le problème de la drogue dans les prisons. y compris sa contribution à la violence en milieu carcéral. était important (Groupe d’étude sur la réduction de la toxicomanie, 1991, p. 12).

Fait plus important encore, le partage d'aiguilles est fréquent et il arrive souvent que de 15 à 20 personnes utilisent une aiguille sans la nettoyer entre chaque usage. Le fait que plus de quinze détenus d'un établissement en Écosse ont contracté une infection à VIH en quelques mois à peine après avoir partagé du matériel d'injection constitue une preuve irrécusable de la nécessité d'éviter ou de réduire les risques que pose la consommation de drogues injectables (renseignements communiqués par Timothy Harding; les résultats de l'étude dans la prison écossaise doivent être publiés en 1994). La consommation de drogue en milieu carcéral n'est pas un problème exclusif au Canada mais un problème universel. Riley a dit récemment que bon nombre de détenus des établissements correctionnels fédéraux et provinciaux intègrent la consommation de drogue à leur mode de vie et qu'aucun incitatif important ne les en empêche, parce que «le retrait des privilèges ne pèse pas lourd dans la balance face aux paradis artificiels» (Riley, 1993, pp. 3-4). Riley a conclu ainsi :

On donne souvent l'impression que les prisons sont un monde à part. En fait. c'est tout le contraire: les prisons connaissent les problèmes de lextérieur et leurs problèmes propres, et des gens circulent constamment d'un univers à l'autre. (Ibid.)

Tout comme dans la société, les efforts visant à interdire la consommation de drogue sont coûteux et pas toujours fructueux. Ces efforts comprennent l'interdiction des drogues. la pénalisation de la possession et de l'usage des drogues, l'éducation et les programmes de traitement. Comme il est irréaliste de croire que tous les détenus cesseront de faire usage de drogues injectables, il est probable que le VIH se propagera implacablement parmi les détenus qui utilisent des drogues injectables jusqu'à ce qu'ils cessent de partager leurs seringues ou qu'ils aient au moins les moyens pour les nettoyer après usage. Les autres solutions à ce problème semblent insuffisantes, voire nuisibles. Augmenter les ressources visant ì éliminer le phénomène serait probablement inutile et aurait pour seul effet d'inciter les utilisateurs de drogues à se cacher davantage et à adopter des pratiques dangereuses. La communication de l'identité des détenus infectés aux pairs serait aussi contre-productive. car elle nuirait aux message transmis touchant l'utilisation plus sécuritaire des seringues, c'est-à-dire le recours à des précautions d'applications universelles.

Étant donné que les efforts déployés pour mettre un terme à la consommation de drogue ont peu de chances d'éliminer le risque de transmission du VIH, que l'usage de drogues injectables est probablement là pour rester et que la divulgation de l'identité des détenus infectés connus n’abolirait 
pas non plus le risque de transmission du VIH, la seule façon réaliste de prévenir la transmission des infections est d'inciter les utilisateurs de drogues injectables à utiliser en tout temps des seringues propres et de leur donner les moyens de le faire ${ }^{19}$. Opter pour cette approche n'équivaut absolument à approuver la consommation de drogue. mais semble nécessaire pour protéger les détenus, le personnel et la population du danger beaucoup plus grand (que l'utilisation de drogues en soi) que représente l'infection à VIH, et cette option peut s'inscrire dans un modèle de santé ou modèle de réduction des dangers liés au VIH/sida et à l'usage de la drogue 20 .

En conclusion, la façon la plus efficace et la moins dommageable de réduire la transmission du VIH par le partage de seringues contaminées par le VIH serait de faire encore davantage pout diminuer les dommages reliés à la consommation de drogue, et ce en offrant plus de programmes de traitement. en éduquant mieux les détenus et en leur donnant accès à des produits de nettoyage des seringues ou en leur fournissant des seringues propres.

19. Le Comité sur le sida et les prisons recommande, entre autres, que de l'cau de Javel concentrée soit mise à la disposition des détenus dans les établissements correctionnels fédéraux et que "des études soient effectuées en vue de trouver des moyens et d'ćlaborer des mesures, notamment l'accès à du matériel d'injection stérile, qui permettront de réduire encore davantage le risque de transmission du VIH et les autres dangers découlant de l'utilisation de drogues injectables dans les établissements correctionnels fédéraux. Ces études devraient être effectuées par des personnes de l'extérieur du SCC, mais en collaboration avec celui-ci, et Santé Canada devrait y participer étroitement, et clles devraient être précédées de consultations auprès des détenus, du personnel, de groupes communautaires et de spécialistes indépendants. Elles devraient comprendre des projets pilotes valides sur le plan scientifique et être alliêes à des mesures de planification, de communication et de sensibilisation qui permettront d'accélérer la mise en ouvre de la distribution de matériel d'injection stérile dans les établissements. »

20. Ce modèle a été défini ainsi dans le contexte de l'usage de la drogue (voir Riley, 1993, p. 5) : « Même si la réduction des dangers vise à réduire la consommation de drogue, elle met en priorité la réduction des conséquences négatives du phénomène [.... Selon celte approche, une stratégie visant exclusivement a diminuer l'usage de la drogue peut avoir pour tout résultat d'augmenter la fréquence de divers dangers liés à la drogue [...]. On tente donc d'atténuer les problèmes et on reconnaît que l'abstinente n'est pas un objectif réaliste ni souhaitable, surtout à court terme. Il ne faut pas en conclure que la réduction des dangers et l'abstinence s'excluent mutueliement, mais plutôt que l'abstinence n'est pas le seul objectif acceptable et imporlant. Selon l'approche de la réduction des dangers, on fixe une hierarchic d'objectils, on incite les intéressés à alteindre les plus immédiats et les plus téalistes, el on les amène progressivement à une consommation sans risque ou, si possible, a l'abstinence. C'est donc une approche qui se caractérise par le pragmatisne [...]. Un certain nombre de pays ont mis au point des stratégies de ce genre après avoir pris conscience que la transmission du sida était une plus grande menace pour la santé individuelle et publique que la consommation de droguc." 


\section{(6) L'intention de blesser ou de transmettre le VIH}

Un détenu peut faire savoir à un autre détenu ou à un employé son intention de blesser quelqu'un ou d' «infecter quelqu'un du VIH». Dans de tels cas, il n'est pas nécessaire de savoir si le détenu est infecté par le VIH pour intervenir afïn de prévenir l'accident. Il faudrait tenter de l'empêcher de mettre ses menaces à exécution. sachant ou non s’il est infecté par le VIH. Cependant, dans certains cas particuliers. on peut être fondé à informer le personnel que le détenu souffre d'une maladie infectieuse et qu'il a menacé de la transmettre. parce qu'il peut être nécessaire de prendre des précautions particulières dans les contacts quotidiens avec ce détenu. $\mathrm{Ce}$ pendant, il n'est pas nécessaire de préciser qu'il est infecté par le VIH.

\section{Divulgation après l'exposition}

L'exposition au VIH suscite presque toujours une peur et une détresse intenses, et on prétend souvent que la personne qui peut avoir été exposée a le droit de connaître l'état sérologique de la personne qui est à l'origine de l'exposition. Encore ici, il faut établir si la divulgation est nécessaire. En général. la personne qui a pu être exposée au VIH doit agir comme si la source de l'exposition était infectée, à moins qu'elle ne soit certaine que ce n'est pas le cas. Il est difficile de s'assurer d'un tel fait. La divulgation de l'état sérologique ne fournit que des renseignements d'une utilité limitée. Elle peut confirmer avec précision que la personne qui a pu exposer autrui était infectée au moment de l'exposition potentielle, mais elle ne peut garantir que la personne n'était pas infectée à ce moment. Dans certains cas. le résultat du test serait négatif même si la personne était infectée et infectieuse (par exemple pendant la «période de latence», avant que les anticorps du VIH ne puissent être détectés). Dans ce cas, la personne qui a pu être exposée serait rassurée, à tort. pensant qu'elle n'a pas été exposée. La divulgation ne garantira pas à la personne qu'elle n'a pas été exposée au VIH. Celle-ci ne devrait donc pas avoir de rapports sexuels non protégés par lesquels elle pourrait transmettre le VIH après une exposition potentielle, peu importe que la personne ayant pu l'exposer ait reçu un résultat négatif ou positif. En outre, on peut se demander si la personne qui a pu être exposée a besoin de ce renseignement pour décider de suivre ou non un traitement à la zidovudine (prophylaxie post-exposition). Pour être le moindrement efficace, le traitement à la zidovudine. dont l'efficacité n'a pas été prouvé (Weiss, 1992; U.S. Department of Health and Human Services, 1990; Lange et coll., 1990; anonyme. 1993; éditorial, 1993), doit commencer le plus tôt possible après une exposition potentielle, et non pas être retardé jusqu’à la divulgation des renseignements.

Compte tenu de l'incertitude des renseignements fournis et du fait que les seules mesures efficaces et fiables dont la personne qui a pu être exposée peut se servir sont l'éducation. le counselling. le soutien et peut-être un 
traitement à la zidovudine, il est peu probable que la divulgation de l'état sérologique de la personne qui est à l'origine de l'exposition soit nécessaire. Pourtant, il y a un cas dans lequel la communication pourrait être justifiée à titre exceptionnel : celui où la personne qui a pu être exposée est enceinte et a besoin de tous les renseignements pouvant lui être utiles pour décider de mettre fín ou non à sa grossesse ${ }^{21}$.

\section{Conclusion}

D'après ce qui précède, il est évident que, dans la majorité des cas, il est peu probable que la communication de renseignements médicaux sur les détenus permette d'atteindre les objectifs pour lesquels elle est demandée, et qu'elle peut même être contre-productive. Comme nous l'avons dit précédemment. les raisons suivantes expliquent cette situation : 1) les renseignements seraient rarement utiles à la protection de la personne, si ce n'est jamais; 2) dans leur propre intérêt, le personnel et les détenus doivent recourir à des précautions universelles et supposer que tout un chacun dans l'établissement est ou peut être infectieux ; 3) il existe des mesures moins envahissantes qui seraient aussi efficaces, sinon plus, que la communication de l'état sérologique.

Les risques et les préjudices résultant de la divulgation sont bien documentés dans la littérature médicale, scientifique et populaire (Chateauvert et coll. 1990). Dans les établissements correctionnels. ces risques et préjudices peuvent être encore plus grands pour de nombreuses raisons. En premier lieu. la mobilité restreinte des prisonniers peut augmenter les risques et préjudices pouvant découler de la connaissance qu'une personne est séropositive (Gilmore et Somerville, 1989). En deuxième lieu, dans une collectivité fermée, la nouvelle que quelqu'un est infecté par le VIH peut se répandre facilement, et il sera donc diffícile pour la personne visée de se protéger contre la stigmatisation. l'isolement ou le blâme ou de ne pas être le bouc émissaire. En troisième lieu, certains des avantages ou services offerts aux personnes infectées par le VIH ou auxquels elles ont accès de façon discrète à l'extérieur de la prison peuvent être interdits, en totalité ou en partie, aux détenus. Mentionnons le counselling par les pairs et leur soutien. les régimes spéciaux et les traitements expérimentaux (Tomasevski, 1991).

21. Lorsque ce renseignement est requis, on devrait demander à la personne qui a pu exposer la ferme enceinte de consentir à divulguer son état sérologique. Si cette personne ne le connaît pas, on devrait lui demander d'accepter de subir un test et d'en communiquer les résultats à la personne exposcé. Si elle refusc de communiquer les renscignements pertinents ou de subir un test et d'en communiquer le résultat, la personne exposéc aura comme scul recours de dentander au tribunal d'ordonner que l'on obtienne ce renseignement el qu'on te lui communique. 
La communication de renseignements médicaux personnels peut ébranler la confiance qu'ont les détenus dans les services de santé de la prison, augmenter leur méfiance envers les autorités carcérales et les dissuader de demander du counselling ou d'avoir recours aux services de santé. Point encore plus important, moins de détenus demanderont d'euxmêmes de subir un test de détection des anticorps anti-VIH ou révéleront être infectés.

\section{Préoccupations particulières relatives à la communication de renseignements médicaux personnels}

Le milieu carcéral suscite des préoccupations particulières relatives à la communication de renseignements médicaux personnels. Certaines ont déjà été discutées, mais elles seront résumées brièvement dans les sections qui suivent.

\section{Communication de renseignements médicaux sur un détenu au personnel}

En règle générale, la prétention selon laquelle le personnel a besoin de connaître les renseignements médicaux confidentiels sur les détenus n'est pas justifiée: les renseignements médicaux sur les détenus, sans le consentement de ces derniers, ne peuvent être communiqués au personnel. Cette règle tolère quelques exceptions lorsque, dans des cas particuliers et après évaluation individuelle du cas, il est établi qu'une telle communication est nécessaire pour empêcher un préjudice grave et qu'il n'y a pas de moyen moins intrusif et moins restrictif permettant d'atteindre le même objectif.

L'application universelle de procédures et de pratiques de protection éliminera le «besoin de savoir» dans la plupart des cas où l'exposition au VIH peut survenir. D'autres situations de ce genre peuvent être évitées si l'on tente de prévenir l'exposition au sang et aux fluides corporels. Point encore plus important, la communication peut quelquefois rassurer à tort le personnel, sans le protéger réellement. Les risques et les préjudices résultant d'une telle communication semblent l'emporter sur les avantages marginaux qu'elle pourrait présenter.

\section{Communication de renseignements médicaux sur un détenu au di- recteur de l'établissement}

La communication de renseignements médicaux au directeur ou au personnel administratif de l'établissement n'est pas en règle générale justifiée. Dans l'exécution de leurs fonctions en vertu de la Loi sur le système correctionnel et la mise en liberté sous condition. les directeurs n'ont pas à connaître l'état sérologique des détenus. Ils doivent plutôt remplir leurs fonctions comme si la population carcérale comportait des détenus infectés par le VIH. Cependant. dans certains cas exceptionnels, la communication 
de renseignements médicaux sur un détenu au directeur de l'établissement peut être justifiée ou même nécessaire. Il en serait ainsi, par exemple, si le personnel médical savait qu'un détenu en particulier a l'intention de blesser quelqu'un d'une façon qui pourrait l'exposer au VIH et que cette personne devait être avertie ou que des mesures de protection devaient être prises. Dans une telle situation, il peut être nécessaire d'informer le directeur de la possibilité d'un tel projet ou de l'activité donnant lieu à un risque. Il suffit alors d'informer le directeur que le détenu souffre d'une «maladie transmissible».

\section{Communication de renseignements médicaux sur un détenu à un agent de gestion des cas et à l'agent de libération conditionnelle.}

Il semble y avoir peu de cas, si tant est qu'il y en ait, où la communication de renseignements médicaux sur un détenu à l'agent de gestion du cas ou à l'agent de libération conditionnelle serait justifiée sans le consentement du détenu.

\section{Communication de renseignements médicaux sur un détenu aux autorités de la collectivité}

Il y a peu de cas, si tant est qu'il y en ait, semble-t-il, où la communication de renseignements médicaux sur un détenu aux autorités de la collectivité serait justifiée sans le consentement du détenu.

\section{Communication de renseignements médicaux sur un détenu à ses pairs}

En général, la prétention selon laquelle les détenus ont besoin de connaître les renseignements médicaux confidentiels sur d’autres détenus n'est pas justifiée. Une telle communication, sans le consentement du détenu, devrait être nécessaire et efficace et constituer le moyen le moins invasif et le moins restrictif possible permettant d'atteindre l'objectif. Il peut se présenter des cas où la communication est justifiée. par exemple la notification du partenaire qui ne se doute de rien. Encore une fois, il s'agit d'une justification ponctuelle, comme dans tous les autres cas.

\section{o. Communication de renseignements médicaux sur un détenu au partenaire qui lui rend visite}

Lorsque le personnel des services de santé de l'établissement sait qu'un détenu est infecté par le VIH, il doit s'assurer que ce détenu n'exposera pas au VIH le partenaire qui lui rend visite et qui ne se doute de rien. Dans un tel cas où il faut avertir le partenaire, le personnel médical doit procéder selon la méthode de recherche des contacts recommandée par le Comité consultatif national sur le sida (Gilmore et Somerville, 1989 , pp. 16-19). On devrait demander au détenu d'informer son partenaire ou de consentir à ce que quelqu' un le fasse pour lui. Cependant, s'il refuse, le personnel médical devrait informer le partenaire avant la visite. ou la visite 
devrait être retardée jusqu'à la notifïcation du partenaire. Le personnel médical devrait rencontrer le détenu que l’on sait infecté ainsi que son partenaire, pour discuter de la façon d'éviter l'exposition au VIH par voie sexuelle et les aider à résoudre les problèmes éventuels. Une telle procédure $s$ 'appliquerait aux détenus ayant une permission de sortir. Lorsque le partenaire ne peut rencontrer le personnel médical, on peut organiser un suivi par l'intermédiaire du médecin de premier recours du partenaire. Tous les détenus. que l'on sache ou non s'ils sont infectés par le VIH, devraient être encouragés à discuter avec leur partenaire de l'exposition au VIH par voie sexuelle.

Lorsqu'une visite est retardée ou annulée. il ne faudrait pas en divulguer la raison au personnel ou aux autres détenus.

\section{Communication de renseignements médicaux sur un membre du personnel aux détenus}

Cette situation est semblable à celle déjà examinée (communication de renseignements médicaux sur un détenu au personnel). Une demande de communication de renseignements ne peut être justifiée que rarement, et seulement dans des cas particuliers.

\section{Communication de renseignements médicaux sur un membre du. personnel aux collègues}

Encore une fois, cette situation est semblable à celle qui vient d'être examinée (communication de renseignements médicaux sur un détenu au personnel). Une demande de communication de renseignements ne peut être justifiée que rarement, et seulement dans des cas particuliers.

\section{Communication de renseignements médicaux à un membre du}

personnel ou à un détenu qui a subi une exposition au sang d'un détenu ou d'un membre du personnel

Cette situation a été examinée en détail ci-dessus (communication après l'exposition potentielle au VIH). Une demande de communication de renseignements médicaux, sans le consentement de la personne dont le sang est la source de l'exposition, ne peut être justifiée que rarement, et seulement dans des cas particuliers. Dans le cas d'une exposition susceptible d'avoir transmis le VIH, des services d'information, de counselling et de soins médicaux devraient être facilement accessibles à la personne exposée, qu'il s'agisse d'un membre du personnel ou d'un détenu. Il a été prétendu que. sans communication des renseignements médicaux à un détenu qui a été blessé par un autre détenu, «il pourrait être impossible de traiter le détenu malade" (Zazulak, 1992: c'est nous qui traduisons). Cependant, il faudrait au contraire envisager diverses options de traitement. que l'on sache ou non si la source de l'exposition est infectée par le VIH. 


\section{Test de dépistage chez les agresseurs sexuels déclarés coupables}

Récemment, un homme est décédé de maladies liées au sida onze semaines après avoir été déclaré coupable d'agression sexuelle sur un garçon de cinq ans. Le coroner de Montréal, Claude Paquin, qui a effectué une autopsie sur le défunt. a recommandé dans son rapport que le SCC et le ministère de la Sécurité publique du Québec étudient la possibilité d'obliger les agresseurs sexuels déclarés coupables à subir un test de détection des anti-corps anti-VIH (Gouvernement du Québec, Bureau du coroner, 1992). Un tel test viserait, a-t-il dit, à prévenir la propagation de l'infection à VIH au sein du système carcéral. d'une part, et à produire des renseignements afin d'informer les victimes d'agression sexuelle si elles ont ou n'ont pas été exposées au VIH, d'autre part ${ }^{22}$. Touchant l'obligation des agresseurs sexuels à passer un test. le Comité consultatif national sur le sida a adopté la résolution suivante :

Le Comité consultatif national sur le sida recommande fortement que la recherche de l'anti-VIH soit volontaire pour les personnes accu sées d'avoir commis une agression sexuelle susceptible de transmettre l'infection à VIH. et que les résultats de l'épreuve soient traités en toute confidentialité exception faite de leur divulgation à la victime.

Le Comité consultatif national sur le sida recommande fortement que la recherche de l'anti-VIH soit volontaire pour les personnes reconnues coupables d'une forme d'agression sexuelle susceptible de transmettre l'infection à VIH, et que les résultats de l'épreuve soient traités en toute confidentialité - exception faite de leur divulgation à la victime. (Somerville et Gilmore, 1988, p. xxii.)

Il ne semble pas justifié d'obliger les personnes reconnues coupables d'une forme d'agression sexuelle a passer un test, car cette mesure n'est ni efficace. ni nécessaire à l'atteinte de l'un des objectifs mentionnés dans le rapport du coroner. Obliger une personne reconnue coupable à passer le test dans le but de protéger la victime et les tiers ou rassurer la victime n'est pas, dans la plupart des cas, nécessaire, puisqu'il s'écoule en général entre huit et douze mois avant qu'une personne accusée d'agression sexuelle soit

22. Ibid. p. 3. Dans un autre cas, une autre question, encore plus complexc, a été soulcvée : laudrait-il faire passer un test de détection des anticorps anti-VIH aux personnes accusćes d'agression scxuclle? Margot B. a accusé un homme qui était a l'extérieur de la prison grâce à un laisser-passer d'une jouméc pour travailler à un projel communautaire de l'avoir violée dans le presbytère de Dunham. Lorsqu'il s'est prononcé sur la demande de faire subir a l'accusé un test de détection des anticorps anti-VIH, le juge Roberge a déclaré qu'un homme accusé de viol ne peut être forcé de subir un tcl test. Depuis, Margot B. a lancé une campagne nationale demandant qu'on oblige toutes les personnes accusées d'agression sexuelle a subir un test de délection des anticorps anti-VIH. Pour une discussion plus détaillée, voir Jürgens ct coll. (1993). 
reconnue coupable. À ce moment-là, la victime peut aller passer elle-même le test et obtenir des renseignements fiables pour savoir si elle a contracté le VIH, alors que les tests de détection subis par l'agresseur ne pourraient fournir ce renseignement. Obliger une personne reconnue coupable à passer le test dans le but de prévenir la transmission du VIH dans les établissements correctionnels n'est pas non plus nécessaire et ne permettrait pas d'atteindre cet objectif. En outre, il existe des mesures moins intrusives. De plus, les détenus infectés qui seraient identifiés après avoir passé le test deviendraient plus vulnérables en prison. Les tests obligatoires appliqués à cette population carcérale seraient discriminatoires et stigmatiseraient les détenus comme une source possible et une menace d'infection dans les prisons. Obliger une personne reconnue coupable à passer le test dans le but de recueillir des renseignements sur son état sérologique afin que ces renseignements puissent être communiqués, le cas échéant, à des victimes éventuelles ne serait pas non plus justifié. Si la personne avait eu un résultat négatif. ceci ne voudrait pas dire qu'elle serait toujours séronégative au moment de la nouvelle agression sexuelle, en raison de la "période de latence» et du temps écoulé entre le moment du test et la nouvelle agression. Par contre, si la personne avait eu un résultat positif, ceci n'apporterait pas beaucoup à la victime non plus. Qu'elle connaisse ou non l'état sérologique de l'agresseur, elle devrait, pour son bien et le bien de ses proches, agir comme si elle avait été exposée au VIH jusqu'au moment où elle saurait si elle a été infectée ou non.

\section{CONCLUSION}

Dans les établissements correctionnels fédéraux, la divulgation de renseignements médicaux personnels sans le consentement de la personne est rarement justifiable. Dans la majorité des cas, une telle communication ne peut être considérée comme nécessaire, et son efficacité est contestable. Elle semble souvent contre-productive ou préjudiciable, et les inconvénients sont de loin supérieurs aux avantages, réels ou possibles, qui pourraient en résulter. Les mesures susceptibles d'être prises afin d'empêcher l'exposition au VIH et l'infection doivent l'être, peu importe si le personnel. le directeur de l'établissement ou les détenus savent ou non si le détenu ou le membre du personnel est infecté ou non par le VIH. Il est essentiel d'informer le personnel et les détenus sur les précautions pouvant prévenir la transmission du VIH et de mettre à leur disposition les moyens nécessaires, si l'on veut prévenir la transmission de l'infection à VIH dans les établissements correctionnels. La divulgation ne sera justifiée que rarement, dans des cas exceptionnels, lorsqu'une évaluation individuelle indique que cette communication est nécessaire, susceptible d'être efficace et qu'elle constitue le moyen le moins intrusif et le moins restrictif possible pour prévenir des préjudices qui ne peuvent l'être autrement. Dans tous les 
autres cas où la divulgation est demandée, d'autres moyens disponibles s'imposent, qui seraient moins dommageables et plus susceptibles d'être efficaces.

\section{BIBLIOGRAPHIE}

AMERICAN MEDICAL ASSOCIATION, Board of Trustees (1987), "Prevention and Control of Acquired Immunodeficiency Syndrome: An Intcrim Report", 258 JAMA 2100.

ANONYME (1992), «HIV Seroconversion After Occupational Exposure Despite Prophylactic Zidovudine Therapy », Lancet, vol. 341, pp. 1077-1078.

ANONYME (1993), "Why Anonymous", Lancet, vol. 341, pp. 1059-1060.

ASSOCIATION MÉDICALE CANADIENNE (1989), Une position de l'AMC.

ASSOCIATION MÉdICALE CANADIENNE (1993), «Sommaire de politique de

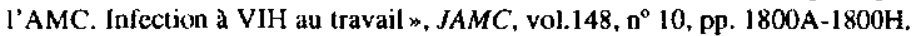

BELITZKY, R., et SOLOMON, R. A. (1987), «Doctors and Patients : Responsibilities in a Confidential Relationship ", in L. H. DALTON et S. BURRIS (dir.), AIDS and the Law: A Guide for the Public, New Haven, Yale University Press, Pp. 201-209, 202.

BREWER, T. F. (1991), «HIV in Prisons: The Pragmatic Approach", AIDS, $\mathrm{n}^{\circ}$ 5, p. 897.

CASSWELL, D. G. (1989), «Disclosure by a Physician of AIDS-related Patient Information : An Ethical and Legal Dilemna ", Revue du Barreau Canadien, pp. 225 258 .

CENTERS FOR DISEASE CONTROL (1987), Update: Human Immunodeficiency Virus Infections in Health Care Workers Exposed to Blood of Infected Patients, Morb Mortal Weekly Rep, $\mathrm{n}^{\circ}$ 36, pp. 285-295.

CHATEAUVERT, M., DUFFIE, A. et GILMORE, N. (1990), Human Immunodeficiency Virus Antibody Testing: Counselling Guidelines from the Canadian Medical Ascociation, Oltawa, Association médicale canadienne, pp. 1-12.

COMITÉ CONSULTATIF NATIONAL SUR LE SIDA (1992), Le VIH et les droits de la personne au Canada.

COMITÉ PARLEMENTAIRE SUR LE SIDA (1990), Le SIDA : un défi.

LE COMMISSAIRE À LA PROTECTION DE LA VIE PRIVÉE (1989), Le SIDA et la Loi sur la Protection des renseignements personnels, Ottawa, Ministre des Approvisionnements et Services Canada.

DECKER, M. D. et coll. (19), «Serocpidemiology of Hepatitis B in Tenoessee Prisons », Journal of Infectious Diseases, $\mathrm{n}^{\prime \prime} 150$, p. 450.

DICKENS. B. M. (1990), "Confidentiality and the Duty w Warn», in L. O. Gostin (dir.), AlDS and the Health Care System, New Haven, Yalc University Press, pp. $98-112$. 
DOYLE, J. (1991), «Management Issues. A Prison Officers Union Perspective», in Norberry et al (dir.), HIVIAIDS and Prisons, Canberra, Australian Institute of Criminology, pp. 125-131.

FORD, P. M., CONNOP, P. J., PANARO, L. et coll. (1993), Seroprevalence of HIV.I in an Ontario Male Medium Security Penitentiary, document non publié.

FRIEDLAND, G., KAHL, P., SALTZMAN, B. et coll. (1990), "Additional Evidence for Lack of Transmission of HIV Infection by Close Interpersonal (Casual) Contact $», A J D S, \mathrm{n}^{\circ} 4$, pp. 639-644.

GERBERDING, J. L., LITTELL, C., TARKINGTON, A. et coll. (1990), «Risk of Exposure of Surgical Personnel to Patients' Blood during Surgery at San Francisco General Hospital ", New England Journal of Medicine, vol. 322, pp. 17881793.

GERSHON, R. R. M., VLAHOV, D. el NELSON, K. E. (1990), «The Risk of Transmission of HIV-1 Through Non-Percutaneous, Non-Sexual Modes. A Review», AIDS, $\mathrm{n}^{\circ} 4$, pp. 645-650.

GILMORE, N., et SOMERVILLE, M. A. (1989), Physicians, Ethics and AIDS, Ottawa, Association médicale canadienne, $\mathbf{n}^{\circ} \mathbf{2 1}$.

GILMORE, N., SOMERVILLE, M. A., JÜRGENS, R., et WILSON, S. (1993), «Is Compulsory Testing of a Person Charged with «Rape» Ever Justified? », Présentation de l'affiche PO-D27-4187 a la IX ${ }^{e}$ Conférence internationale sur le SIDA, Berlin, du 7 au 11 juin.

GOUVERNEMENT DU QUÉBEC, BUREAU DU CORONER (1992), Rapport d'investigation, Claude Paquin, coroner investigateur, Montréal, le 24 novembre 1992.

Groupe de travail fédéral-provincial sur la confidentialité concemant la séropositivité à l'égard du VIH (1988), Santé et Bien-Être social Canada, novernbre.

Groupe d'étude sur la réduction de la toxicomanie (1991), Ottawa, Approvisionnement et Services Canada.

HAMBLIN, J., DUCKETT, M., SOMERVILLE, M. A., GILMORE, N. et ZIMMERMAN, S. (1990), Responding to HIV/AIDS in Canada, Carswell, Toronto.

HANKINS, C.. et coll. (1989), «Infection au VIH-1 dans une prison à sécurité intermédiaire pour fenmes. Québec», Rapport hebdomadaire des maladies au Canada, $\mathrm{n}^{\circ} 168$, pp. 15-33.

HARDING, T. (1990), «HIV Infection and AIDS in the Prison Environment : A Test Case for the Respect of Human Rights », in J. Strang, G. Stimson (dir.), AIDS and Drug Misuse, New York, Routledgc, pp. 197-207.

HEILPERN, H. et EGGER, S. (1989), AWS in Australian Prisons. Issues and Policy Options, Canberra, Department of Community Services and Health.

HOUGH, A. el SCHWARTZ, D. M. (1985), Aids and Prisons: Working our Way out of the Maze, document présenté dans le cadre de l'Industrial Issues Workshop. Conlerence nationale [australienne] sur le SIDA.

JÜRGENS, R., GILMORE, N. et SOMERVILLE, M. A. (1993), "Are Compulsory HIV Testing and Disclosure of HIV Status Ever Justifiable? Ethical and Legal Analysis Using Sex Offenders, Health Care Providers, Patients as Examples». présentation d'une affiche a la Troisième conférence annuelle de ki recherche sur le VIH/sida, Montréal, du 13 au 15 nuai, Joturnal canadien des maladies infecfieu.ses, $\mathrm{n}^{\prime \prime}$ 4, (suppl. B), 48 B. SP-403. 
LABORATOIRE DE LUTTE CONTRE LA MALADIE (1992), "Risques de transmission des pathogènes à diffusion hématogènes dans les établissements de soins de santé ", Relevé des maladies transmissibles au Canada, $\mathrm{n}^{\circ}$ 18, pp. 177-184, 178.

LANGE, J. M. A., BOUCHER, C. A. B., HOLLAK, C. E. M. et coll. (1990), «Failure of Zidovudine Prophylaxis After Accidental Exposure to HIV-1 ", New England Journal of Medicine, vol. 322. pp. 1375-1377.

LIFSON, A. R. (1988), « Do Alternate Modes for Transmission of Human Immunodeficicncy Virus Exist?», JAMA, n' 259, pp. 1353-1356.

MIKE, L., OSTROWSKY, J., BEHENY, J. et HEWITT, M. (1991), «HIV in the Health Care Workplace», Washington, Office of Technology Assessment, Congress of the United States of America.

NACCI, P., et KANE, T. (1982), Sex and Sexual Aggression in Federal Prisons. Washington, Federal Burcau of Prisons, pp. 7-9.

NEVELOFF DUbleR, N., BERGMAN, C. M., FRANKEL, M. E. et coll. (1990), «Management of HIV Infection in New York State Prisons», Columbia Human Rights Law Review, $\mathrm{n}^{\circ} 21$, pp. 363-400.

PICARD. E. I. (1984), Legal Liability of Doctors and Hospitals in Canada, $2^{\mathrm{e}}$ ed.

RICHMAN, K. M., et RICKMAN, L. S. (1993), «The Potential for Transmission of Human Immunodeficiency Virus Through Human Bites », J Acq Immune Defic Synd, $\mathrm{n}^{\circ}$ 6, pp. 402-406.

RILEY, D, (1993), «Drug Use in Prisons : A Harm Reduction Approach», mémoire présenté au CESP, janvier.

ROGERS, D. E. et GELLIN, B. G. (1990), commentaire de la rédaction, « The bright spot about AIDS : it is very tough to catch », AIDS, n 4, pp. 695-696.

SANTÉ ET BIEN-ÊTRE SOCIAL CANADA (1987), "Recommandations visant a prévenir la transmission du VIH en milieu de soins ", Rapport hebdomadaire des maladies au Canada, 13 S3, pp. 1-10.

SERVICE CORRECTIONNEL DU CANADA (1990), Direction de la recherche et de la statistique, Conclusions de l'Enquête nationale sur l'alcool et les autres drogues, Forum. Recherche sur l'achualité correctionnelle, Ottawa, Service correctionnel du Canada, vol. $2, n^{\circ} 4$, pp. 3-6.

SHAW, S. (1988), "Prisoners' Rights", in P. Sieghart (dir.), Human Rights in the United Kingdom, Londres, Pinter Publishers, pp. 40-49.

SOMERVILLE, M. A. (1989), « AIDS : A Challenge to Health Care, Law, and Ethics», in D. Snowden et D. F. Cassidy (dir.), AIDS : A Handbook for Professionals. pp. 200-220.

SOMERVILLE, M. A. ct ORKIN, A. J. (1989), «Human Rights, Discrimination and AIDS : Concepts and Issues $n, A I D S, n^{\circ} 3$, pp. S283-S287.

SOMERVILLE, M. A. et GILMORE, N. (1988), Recherche de l'anticorps contre le virus de l'inimunodéficience humaine au Canada, rapport présenté au Comité consultatif national sur le sida et approuvé par celui-ci, Montréal, Centre de médecine, d'éthique et de droit de l'université McGill, $\mathrm{n}^{\circ} 53 ;$ Rappon hebdomadaire des maladies au Canada-(1989), vol. 15, n², pp. 37-47.

THOMAS, P. A. (1990), "HIV/AIDS in Prisons», The Howard Journal of Criminal Justice, vol. 29, pp. 1-13.

TOMASEVSKY, K. (1991), «AIDS in Prisons », AIDS, vol. 5 (suppl. 1), pp. S245S251. 
TORRE, D., SAMPIETRO, C., FERRARO, G., ZEROLI, C. et SPERANZA, L. (1990), "Letter to the Editor : Iransmission of HIV-1 infection via a sports injury", New Englend Journal of Medicine, vol. 335, p. 1105.

TSOUKAS, C. M., HADJIS, T., SHUSTER, J., THEBERGE, L., FEORINO, P. et O'SHAUGHNESSY, M. (1988), "Lack of Transmission of Hurman Immunodeficiency Virus (HIV) by Bites and Scratches $», J$ Acq Immune Defic Sind, $\mathrm{n}^{\circ} \mathrm{I}, \mathrm{pp}$. 505-507.

U.S. DEPARTMENT OF HEALTH AND HUMAN SERVICES, PUBLIC HEALTH SERVICE (1990), «Public Health Service Statement on Management of Occupational Exposure to Human Immunodeficiency Virus, Including Considerations Regarding Zidovudine Postexposure Use», Morb Mortal Weekly Rep, n³9, RR1, pp. 1-14.

WEISS, S. H. (1992), "HIV Infection and the Health Care Worker», Med Clin N Am, vol. 76, pp. 269-280.

ZAZULAK, M. H. (1992), avocat général, Service correctionnel du Canada, note de service au Dr J. H. Roy, directeur général, Services de santé, le 13 juillet 1992, sur la communication de renseignements médicaux sur les détenus - VIH, etc. - le besoin de savoir. 